\title{
Two-real-scalar-singlet extension of the SM: LHC phenomenology and benchmark scenarios
}

\author{
Tania Robens $^{1, \mathrm{a}}$, Tim Stefaniak ${ }^{2, \mathrm{~b}}$, Jonas Wittbrodt ${ }^{2, \mathrm{c}}{ }_{(\mathbb{C}}$ \\ ${ }^{1}$ Ruder Boskovic Institute, Bijenicka cesta 54, 10000 Zagreb, Croatia \\ ${ }^{2}$ DESY, Notkestraße 85, 22607 Hamburg, Germany
}

Received: 12 September 2019 / Accepted: 15 January 2020 / Published online: 19 February 2020

(C) The Author(s) 2020

\begin{abstract}
We investigate the LHC phenomenology of a model where the Standard Model (SM) scalar sector is extended by two real scalar singlets. A $\mathbb{Z}_{2} \otimes \mathbb{Z}_{2}^{\prime}$ discrete symmetry is imposed to reduce the number of scalar potential parameters, which is spontaneously broken by the vacuum expectation values of the singlet fields. As a result, all three neutral scalar fields mix, leading to three neutral CP-even scalar bosons, out of which one is identified with the observed Higgs boson at $125 \mathrm{GeV}$. We explore all relevant collider signatures of the three scalars in this model. Besides the single production of a scalar boson decaying directly to SM particle final states, we extensively discuss the possibility of resonant multi-scalar production. The latter includes decays of the produced scalar boson to two identical scalars ("symmetric decays"), as well as to two different scalars ("asymmetric decays"). Furthermore, we discuss the possibility of successive decays to the lightest scalar states ("cascade decays"), which lead to experimentally spectacular three- and fourHiggs final states. We provide six benchmark scenarios for detailed experimental studies of these Higgs-to-Higgs decay signatures.
\end{abstract}

\section{Introduction}

The Large Hadron Collider (LHC) at CERN is the first experimental facility that directly probes the mechanism of electroweak symmetry breaking (EWSB), described in the Standard Model of particle physics (SM) by the Brout-EnglertHiggs mechanism [1-6]. The milestone discovery of a Higgs boson with a mass of $\sim 125 \mathrm{GeV}$ in $2012[7,8]$ and the ongoing measurements of its properties at the LHC (see e.g. Ref. [9]) open the door to a deeper understanding of the structure

\footnotetext{
a e-mail: trobens@irb.hr

be-mail: tim.stefaniak@desy.de

c e-mail: jonas.wittbrodt@desy.de (corresponding author)
}

of EWSB. Indeed, this experimental endeavor may reveal first signs of new physics beyond the SM (BSM), as many well-motivated BSM extensions affect the phenomenology of the observed scalar particle. However, by the end of RunII of the LHC, with the full collected data of $\sim 150 \mathrm{fb}^{-1}$ per experiment (ATLAS and CMS) still being analyzed, Higgs signal rate measurements in various production and decay channels [10-20] are so far in very good agreement with the SM predictions.

Extensions of the SM by scalar singlets are among the simplest possible model beyond the SM (BSM). The most general extension of the SM by $n$ real scalar singlet fields $\phi_{i}$ $(i \in[1, \ldots, n])$ has a scalar potential of the form

$V\left(\phi_{i}, \Phi\right)=V_{\text {singlets }}\left(\phi_{i}, \Phi\right)+V_{\mathrm{SM}}(\Phi)$,

where

$$
\begin{aligned}
& V_{\text {singlets }}\left(\phi_{i}, \Phi\right) \\
& \quad=a_{i} \phi_{i}+m_{i j} \phi_{i} \phi_{j}+T_{i j k} \phi_{i} \phi_{j} \phi_{k}+\lambda_{i j k l} \phi_{i} \phi_{j} \phi_{k} \phi_{l} \\
& \quad+T_{i H H} \phi_{i}\left(\Phi^{\dagger} \Phi\right)+\lambda_{i j H H} \phi_{i} \phi_{j}\left(\Phi^{\dagger} \Phi\right)
\end{aligned}
$$

with real coefficient tensors. Here, $\Phi$ describes the scalar $S U(2)_{L}$ doublet field of the SM and $V_{\mathrm{SM}}$ denotes the scalar potential of the SM. An extension by complex singlets can always be brought into this form by expanding fields and coefficients into real and imaginary parts. Since the $\phi_{i}$ are pure gauge singlets they have trivial kinetic terms that do not induce any gauge interactions, leading to the following contributions to the electroweak (EW) Lagrangian:

$\mathcal{L}_{\mathrm{EW}} \supset\left(D^{\mu} \Phi\right)^{\dagger} D_{\mu} \Phi+\sum_{i} \partial^{\mu} \phi_{i} \partial_{\mu} \phi_{i}-V$.

In addition, it is not possible to write down gauge invariant and renormalizable interactions between a scalar singlet and any of the SM fermions. The singlets will therefore only interact with the SM Higgs boson through the couplings of 
the scalar potential and, if they acquire a non-zero vacuum expectation value (vev), mix with the SM Higgs boson and thereby inherit some of its gauge and Yukawa couplings.

This is also the reason why - as long as no new interactions of the scalar singlet fields with additional particles occur - there is no physical difference between a parametrization in terms of $N$ complex scalar singlet fields or $2 N$ real scalar singlet fields. Naively, one would expect that imaginary parts of complex scalar fields are CP-odd, and mixing them with the real parts or the SM Higgs boson would lead to $\mathrm{CP}$-violation. However, due to the singlets not having any gauge or fermion couplings it is always possible to find a CPtransformation under which all of them are CP-even $[21,22]$. Thus any pure singlet extension of the SM is a theory of only CP-even scalars.

Singlet extensions of the SM have been subject to detailed phenomenological studies before. This includes both extensions by a single real singlet [23-27] (see Refs. [28-32] for recent phenomenological studies) and by a complex singlet or two real singlets [33-42]. The models are also interesting in the context of scalar singlet dark matter [43-52] and a strong first-order electroweak phase transition $[48,50,52-$ 57]. We will focus on a specific extension of the SM by two real singlets that has not been previously considered in the literature.

Experimentally, singlet extensions can be explored in two complementary ways at the LHC. First, precise measurements of the $125 \mathrm{GeV}$ Higgs signal rates probe the structure of the doublet-singlet mixing, as well as possible new decay modes of the observed Higgs boson to new light scalar states. Second, direct searches for new scalars may reveal the existence of the mostly singlet-like Higgs bosons. For the latter the discovery prospects depend on the singlet-doublet mixing and the new scalar's mass (both governing the production rates), and on the decay pattern of the produced scalar state. In general, decays directly to SM particle final states as well as to two lighter scalar states ("Higgs-to-Higgs decays") are possible. While some of the former decays are already searched for by the LHC experiments, current search es for Higgs-to-Higgs decays focus almost exclusively on the signatures $h_{S} \rightarrow h_{125} h_{125}$ (where $h_{S}$ denotes the new Higgs state with mass above $250 \mathrm{GeV}$ ) [58-69], or $h_{125} \rightarrow h_{S} h_{S}$ (with the $h_{S}$ mass below $62.5 \mathrm{GeV}$ ) [70-76]. The model considered in the following, however, features also Higgs decays to unidentical scalar bosons ("asymmetric decays"), Higgs decays involving only non-SM-like Higgs bosons, as well as the possibility of successive Higgs-to-Higgs cascade decays. All of these decay signatures have not been experimentally explored in detail yet. ${ }^{1}$ We will extensively discuss them in

\footnotetext{
${ }_{1}^{1}$ A first search result for a symmetric Higgs-to-Higgs decay involving only non-SM Higgs states has been presented by ATLAS in the $W^{+} W^{-} W^{+} W^{-}$final state [61].
}

this paper and show that they lead to novel collider signatures with sizable signal rates that are experimentally interesting for the analysis of Run-II data as well as the upcoming LHC runs. We provide six dedicated two-dimensional benchmark scenarios, each highlighting a different Higgs-to-Higgs decay signature that has not been probed experimentally. We strongly encourage the experimental collaborations to investigate these novel signatures using current and future collider data.

This paper is structured as follows. We introduce the model in Sect. 2 and summarize all relevant theoretical and experimental constraints on the parameter space in Sect. 3 . In Sect. 4 we discuss the collider signatures of the model and present the impact of current LHC searches on the parameter space. In Sect. 5 we propose six benchmark scenarios for LHC searches for Higgs-to-Higgs decay signatures. We conclude in Sect. 6.

\section{The two real singlet model}

\subsection{Scalar potential and model parameters}

The two real singlet model (TRSM) adds two real singlet degrees of freedom to the SM. These are written as two real singlet fields $S$ and $X$. In order to reduce the number of free parameters two discrete $\mathbb{Z}_{2}$ symmetries

$$
\begin{aligned}
& \mathbb{Z}_{2}{ }^{S}: S \rightarrow-S, X \rightarrow X, \mathrm{SM} \rightarrow \mathrm{SM}, \\
& \mathbb{Z}_{2}{ }^{X}: X \rightarrow-X, S \rightarrow S, \mathrm{SM} \rightarrow \mathrm{SM}
\end{aligned}
$$

are introduced. The most general renormalizable scalar potential invariant under the $\mathbb{Z}_{2}{ }^{S} \otimes \mathbb{Z}_{2}{ }^{X}$ symmetry is

$$
\begin{aligned}
V= & \mu_{\Phi}^{2} \Phi^{\dagger} \Phi+\lambda_{\Phi}\left(\Phi^{\dagger} \Phi\right)^{2} \\
& +\mu_{S}^{2} S^{2}+\lambda_{S} S^{4}+\mu_{X}^{2} X^{2}+\lambda_{X} X^{4} \\
& +\lambda_{\Phi S} \Phi^{\dagger} \Phi S^{2}+\lambda_{\Phi X} \Phi^{\dagger} \Phi X^{2}+\lambda_{S X} S^{2} X^{2}
\end{aligned}
$$

All coefficients in Eq. (5) are real, thus the scalar potential contains nine model parameters in total. We provide a translation of these coefficients to the scalar potential parameters in the complex scalar singlet parametrization in Appendix A.

Depending on the vevs acquired by the scalars different phases of the model can be realized. We decompose the fields (in unitary gauge) as

$$
\Phi=\left(\begin{array}{c}
0 \\
\frac{\phi_{h}+v}{\sqrt{2}}
\end{array}\right), \quad S=\frac{\phi_{S}+v_{S}}{\sqrt{2}}, \quad X=\frac{\phi_{X}+v_{X}}{\sqrt{2}}
$$

leading to the tadpole equations

$$
-v \mu_{\Phi}^{2}=v^{3} \lambda_{\Phi}+\frac{v v_{S}^{2}}{2} \lambda_{\Phi S}+\frac{v v_{X}^{2}}{2} \lambda_{\Phi X},
$$




$$
\begin{aligned}
& -v_{S} \mu_{S}^{2}=v_{S}^{3} \lambda_{S}+\frac{v^{2} v_{S}}{2} \lambda_{\Phi S}+\frac{v_{S} v_{X}^{2}}{2} \lambda_{S X}, \\
& -v_{X} \mu_{X}^{2}=v_{X}^{3} \lambda_{X}+\frac{v^{2} v_{X}}{2} \lambda_{\Phi X}+\frac{v_{S}^{2} v_{X}}{2} \lambda_{S X} .
\end{aligned}
$$

These have solutions for any values of $v, v_{S}, v_{X}$. However, to achieve electroweak symmetry breaking $v=v_{\mathrm{SM}} \approx$ $246 \mathrm{GeV}$ is required. If $v_{S}, v_{X} \neq 0$ the $\mathbb{Z}_{2}$ symmetries are spontaneously broken, and the fields $\phi_{h, S, X} \operatorname{mix}$ into three physical scalar states. This is called the broken phase.

If $v_{X}=0$ the field $\phi_{X}$ does not mix with $\phi_{h, S}$, does not acquire any couplings to SM particles, and is stabilized by the $\mathbb{Z}_{2}{ }^{X}$ symmetry. ${ }^{2}$ This makes it a candidate particle for dark matter (DM). The phenomenology of the two visible scalar states is very similar to the real singlet extension [2832]. If both singlet vevs vanish, $\phi_{h}$ is the SM Higgs boson, and the two singlets both form separate dark sectors stabilized by their respective $\mathbb{Z}_{2}$ symmetries. In this case collider phenomenology is (at tree-level) only impacted by possible invisible decays of $h_{125}$ to the DM particles.

In this work, we focus on the broken phase as it leads to the most interesting collider phenomenology. The mass eigenstates $h_{1,2,3}$ are related to the fields $\phi_{h, S, X}$ through the $3 \times 3$ orthogonal mixing matrix $R$

$$
\left(\begin{array}{l}
h_{1} \\
h_{2} \\
h_{3}
\end{array}\right)=R\left(\begin{array}{l}
\phi_{h} \\
\phi_{S} \\
\phi_{X}
\end{array}\right) \text {. }
$$

We assume the mass eigenstates to be ordered by their masses

$M_{1} \leq M_{2} \leq M_{3}$

and parametrize the mixing matrix $R$ by three mixing angles $\theta_{h S}, \theta_{h X}, \theta_{S X}$. Using the short-hand notation

$s_{1} \equiv \sin \theta_{h S}, \quad s_{2} \equiv \sin \theta_{h X}, \quad s_{3} \equiv \sin \theta_{S X}, \quad c_{1} \equiv \cos \theta_{h S}, \ldots$

it is given by

$R=\left(\begin{array}{ccc}c_{1} c_{2} & -s_{1} c_{2} & -s_{2} \\ s_{1} c_{3}-c_{1} s_{2} s_{3} & c_{1} c_{3}+s_{1} s_{2} s_{3} & -c_{2} s_{3} \\ c_{1} s_{2} c_{3}+s_{1} s_{3} & c_{1} s_{3}-s_{1} s_{2} c_{3} & c_{2} c_{3}\end{array}\right)$

where the angles $\theta$ can be chosen to lie in

$-\frac{\pi}{2}<\theta_{h S}, \theta_{h X}, \theta_{S X}<\frac{\pi}{2}$

without loss of generality. In the TRSM it is possible to express the nine parameters of the scalar potential through

\footnotetext{
${\overline{2} \text { The case of } v_{S}}=0$ is equivalent by renaming $S \longleftrightarrow X$.
}

the three physical Higgs masses, the three mixing angles, and the three vevs. These relations are given by

$$
\begin{aligned}
& \lambda_{\Phi}=\frac{1}{2 v^{2}} m_{i}^{2} R_{i 1}^{2}, \quad \lambda_{S}=\frac{1}{2 v_{S}^{2}} m_{i}^{2} R_{i 2}^{2}, \\
& \lambda_{X}=\frac{1}{2 v_{X}^{2}} m_{i}^{2} R_{i 3}^{2}, \\
& \lambda_{\Phi S}=\frac{1}{v v_{S}} m_{i}^{2} R_{i 1} R_{i 2}, \quad \lambda_{\Phi X}=\frac{1}{v v_{X}} m_{i}^{2} R_{i 1} R_{i 3}, \\
& \lambda_{S X}=\frac{1}{v_{S} v_{X}} m_{i}^{2} R_{i 2} R_{i 3},
\end{aligned}
$$

where a sum over $i$ is implied. Fixing one of the Higgs masses to the mass of the observed Higgs boson, $M_{a} \simeq$ $125 \mathrm{GeV}$, and fixing the Higgs doublet vev to its $\mathrm{SM}$ value, $v \simeq 246 \mathrm{GeV}$, leaves seven free input parameters of the TRSM:

$M_{b}, M_{c}, \theta_{h S}, \theta_{h X}, \theta_{S X}, v_{S}, v_{X}$

with $a \neq b \neq c \in\{1,2,3\}$.

This is an important practical difference between the TRSM and another well-studied extension of the SM with two real singlet degrees of freedom, the CxSM [35,38]. The CxSM is expressed in terms of a complex singlet with a softly broken $U$ (1) symmetry of the singlet phase imposed on the scalar potential. This more stringent symmetry leaves only seven model parameters such that one of the physical scalar masses and one of the singlet vevs are dependent parameters. In contrast, the TRSM is consistent for any combination of masses, mixing angles, and vevs, and therefore allows to cover the full possible kinematic phase space of Higgs-toHiggs decay signatures, as we will do when defining the benchmark scenarios in Sect. 5 .

As in all pure singlet extensions the couplings of the scalar boson $h_{a}(a \in\{1,2,3\})$ to all SM particles are given by the $\mathrm{SM}$ prediction rescaled by a common factor

$\kappa_{a}=R_{a 1}$,

where $R_{a 1}$ denotes the doublet admixture of the mass eigenstate $h_{a}$. Due to the orthogonality of the mixing matrix these obey the important sum rule

$\sum_{a=1}^{3} \kappa_{a}^{2}=1$

\subsection{Collider phenomenology}

The triple Higgs couplings are of special phenomenological interest in the TRSM. Using Eq. (15) they can be expressed directly through the input parameters of Eq. (16). The cou- 
pling $\tilde{\lambda}_{a b b}$ of $h_{a} h_{b} h_{b}$ is defined through

$V \supset \frac{h_{a} h_{b}^{2}}{2}\left(\sum_{j} \frac{R_{a j} R_{b j}^{2}}{v_{j}}\right)\left(M_{a}^{2}+2 M_{b}^{2}\right) \equiv \frac{1}{2} \tilde{\lambda}_{a b b} h_{a} h_{b}^{2}$.

Similarly, the coupling of three different scalars is given by

$$
\begin{aligned}
V \supset & h_{a} h_{b} h_{c}\left(\sum_{j} \frac{R_{a j} R_{b j} R_{c j}}{v_{j}}\right) \\
& \times\left(\sum_{i} M_{i}^{2}\right) \equiv \tilde{\lambda}_{a b c} h_{a} h_{b} h_{c},
\end{aligned}
$$

and the triple Higgs self-coupling $\tilde{\lambda}_{a a a}$ reads

$V \supset \frac{h_{a}^{3}}{2}\left(\sum_{j} \frac{R_{a j}^{3}}{v_{j}}\right) M_{a}^{2} \equiv \frac{1}{3 !} \tilde{\lambda}_{a a a} h_{a}^{3}$.

With these definitions the tree-level partial decay width of a scalar $h_{a}$ into two scalars $h_{b}$ and $h_{c}$ (where $b=c$ is allowed) is then given by

$$
\begin{aligned}
\Gamma_{a \rightarrow b c}= & \frac{\tilde{\lambda}_{a b c}^{2}}{16 \pi M_{a}^{3}} \sqrt{\lambda\left(M_{a}^{2}, M_{b}^{2}, M_{c}^{2}\right)} \\
& \times \frac{1}{1+\delta_{b c}} \Theta\left(M_{a}-M_{b}-M_{c}\right),
\end{aligned}
$$

with

$\lambda\left(x_{1}, x_{2}, x_{3}\right) \equiv \sum_{i} x_{i}^{2}-\sum_{i, j \neq i} x_{i} x_{j}$

With this information, the phenomenology of a TRSM Higgs boson $h_{a}$ can be fully obtained from the predictions for a SM-like Higgs boson $h_{\mathrm{SM}}$ of the same mass. Throughout this work we employ the narrow width approximation to factorize production cross sections and branching ratios (BRs).

For a certain production process (e.g. gluon gluon fusion) the cross section, $\sigma$, for $h_{a}$ with mass $M_{a}$ can be obtained from the corresponding SM Higgs production cross section, $\sigma_{\mathrm{SM}}$, by simply rescaling

$\sigma\left(M_{a}\right)=\kappa_{a}^{2} \cdot \sigma_{\mathrm{SM}}\left(M_{a}\right)$

Since $\kappa_{a}$ rescales all Higgs couplings to SM particles, Eq. (24) is exact up to genuine EW corrections involving Higgs self-interactions. In particular, this holds to all orders in QCD.

The scaling factor $\kappa_{a}$ also rescales universally the partial widths of $h_{a}$ decays into SM particles, which in turn leads to a rescaling of the SM total width as

$\Gamma\left(h_{a} \rightarrow \mathrm{SM} ; M_{a}\right)=\kappa_{a}^{2} \cdot \Gamma_{\mathrm{tot}}\left(h_{\mathrm{SM}} ; M_{a}\right)$,

where $\Gamma\left(h_{a} \rightarrow \mathrm{SM} ; M_{a}\right)$ denotes the sum of all partial widths of $h_{a}$ into SM particle final states. Note that this alone can never change the BR predictions of $h_{a}$ into SM particles. Using the results of Eq. (22) we can obtain the BRs of $h_{a}$ decays to other scalar bosons, $h_{a} \rightarrow h_{b} h_{c}$ :

$\mathrm{BR}\left(h_{a} \rightarrow h_{b} h_{c}\right)=\frac{\Gamma_{a \rightarrow b c}}{\kappa_{a}^{2} \Gamma_{\mathrm{tot}}\left(h_{\mathrm{SM}}\right)+\sum_{x y} \Gamma_{a \rightarrow x y}}$.

Denoting the sum of these "new physics" (NP) decay rates to scalar boson final states as

$\mathrm{BR}\left(h_{a} \rightarrow \mathrm{NP}\right) \equiv \sum_{b, c} \mathrm{BR}\left(h_{a} \rightarrow h_{b} h_{c}\right)$,

the BRs of $h_{a}$ decays into any final state $F_{\mathrm{SM}}$ composed entirely of SM fermions and gauge bosons are given by

$\mathrm{BR}\left(h_{a} \rightarrow F_{\mathrm{SM}}\right)=\left(1-\mathrm{BR}\left(h_{a} \rightarrow \mathrm{NP}\right)\right) \mathrm{BR}\left(h_{\mathrm{SM}} \rightarrow F_{\mathrm{SM}}\right)$

One important special case is that in the absence of BSM decay modes — which is always the case for the lightest Higgs bosons $h_{1}-h_{a}$ has BRs identical to a SM-like Higgs boson of the same mass.

Figure 1 shows the decay branching ratios of a SM-like Higgs boson $h_{\mathrm{SM}}$ as a function of its mass. As long as $\mathrm{BR}\left(h_{a} \rightarrow \mathrm{NP}\right)=0$, i.e. if no Higgs-to-Higgs decays are possible for $h_{a}$, the scalar boson $h_{a}$ has exactly the BRs shown in Fig. 1. The numerical values are taken from Ref. [77], based on state-of-the-art evaluations using HDECAY [78-80] and Profecy4F [81-83].

\section{Setup of the parameter scan}

In order to assess the phenomenologically viable regions of the parameter space we apply all relevant theoretical and experimental constraints, which are discussed in the following. We furthermore provide details of our numerical setup.

\subsection{Theoretical constraints}

Unitarity constraints provide important upper bounds on the multi-scalar couplings and the scalar masses. In the TRSM we have derived perturbative unitarity bounds in the high energy limit by requiring the eigenvalues $M^{i}$ of the 2-to-2 


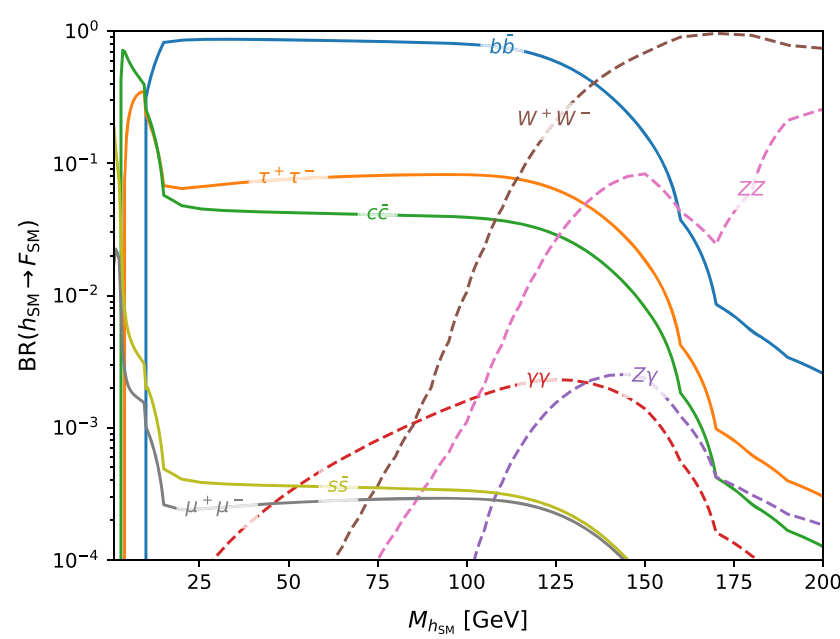

(a) low mass

Fig. 1 Decay branching ratios of a SM-like Higgs boson, $h_{\mathrm{SM}}$, for various SM particle final states, $F_{\mathrm{SM}}$, as a function of its mass, $M_{h_{\mathrm{SM}}}$, in the mass range from $1 \mathrm{GeV}$ to $1 \mathrm{TeV}$, split into the low mass region (left

scalar scattering matrix $M$ to fulfill

$\left|M^{i}\right|<8 \pi$

The resulting constraints on the parameters of the scalar potential are

$\left|\lambda_{\Phi}\right|<4 \pi$

$\left|\lambda_{\Phi S}\right|,\left|\lambda_{\Phi X}\right|,\left|\lambda_{S X}\right|<8 \pi$,

$\left|a_{1}\right|,\left|a_{2}\right|,\left|a_{3}\right|<16 \pi$,

where $a_{1,2,3}$ are the three real roots of the cubic polynomial

$$
\begin{aligned}
P(x) \equiv & x^{3}+x^{2}\left(-12 \lambda_{\Phi}-6 \lambda_{S}-6 \lambda_{X}\right) \\
& +x\left(72 \lambda_{\Phi}\left(\lambda_{S}+\lambda_{X}\right)-4\left(\lambda_{\Phi S}^{2}+\lambda_{\Phi X}^{2}\right)\right. \\
& \left.+36 \lambda_{S} \lambda_{X}-\lambda_{S X}^{2}\right) \\
& +12 \lambda_{\Phi} \lambda_{S X}^{2}+24 \lambda_{\Phi S}^{2} \lambda_{X}+24 \lambda_{\Phi X}^{2} \lambda_{S} \\
& -8 \lambda_{\Phi S} \lambda_{\Phi X} \lambda_{S X}-432 \lambda_{\Phi} \lambda_{S} \lambda_{X} .
\end{aligned}
$$

Closed form conditions for boundedness of the scalar potential, Eq. (5), have been derived in $[84,85]$. In our notation they read

$$
\begin{aligned}
& \lambda_{\Phi}, \lambda_{S}, \lambda_{X}>0 \\
& \bar{\lambda}_{\Phi S} \equiv \lambda_{\Phi S}+2 \sqrt{\lambda_{\Phi} \lambda_{S}}>0 \\
& \bar{\lambda}_{\Phi X} \equiv \lambda_{\Phi X}+2 \sqrt{\lambda_{\Phi} \lambda_{X}}>0 \\
& \bar{\lambda}_{S X} \equiv \lambda_{S X}+2 \sqrt{\lambda_{S} \lambda_{X}}>0 \\
& \sqrt{\lambda_{S}} \lambda_{\Phi X}+\sqrt{\lambda_{X}} \lambda_{\Phi S}+\sqrt{\lambda_{\Phi}} \lambda_{S X} \\
& \quad+\sqrt{\lambda_{\Phi} \lambda_{S} \lambda_{X}}+\sqrt{\bar{\lambda}_{\Phi S} \bar{\lambda}_{\Phi X} \bar{\lambda}_{S X}}>0 .
\end{aligned}
$$

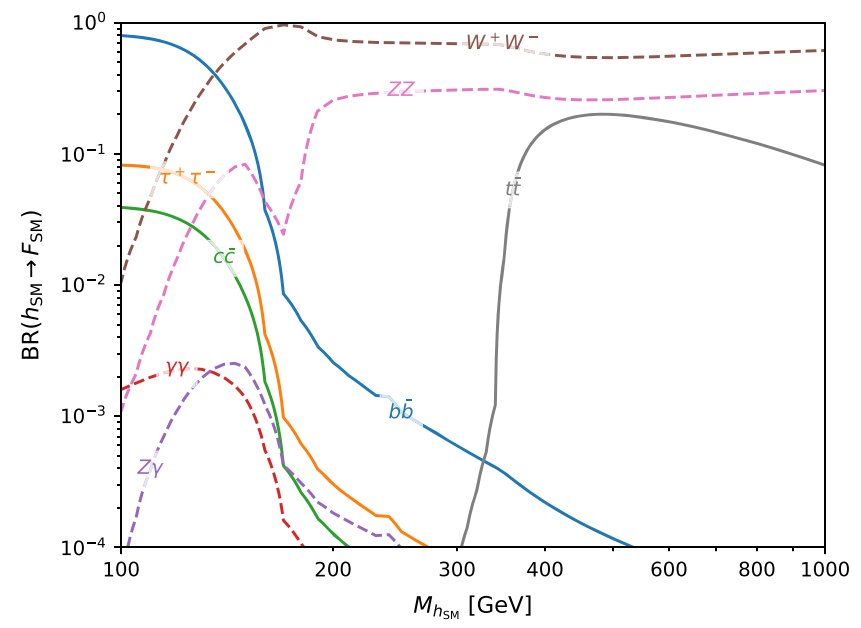

(b) high mass

panel) and the high mass region (right panel). The numerical values are taken from Ref. [77], see text for further details

It has been proven in Ref. [39] that at tree-level a vacuum of the form of Eq. (6) is always the global minimum of the scalar TRSM potential. Therefore no additional constraints from vacuum decay need to be considered.

\subsection{Experimental constraints}

We use the oblique parameters $S, T$ and $U$ [86-89] to parametrize constraints from electroweak precision measurements, which are compared to the latest fit results [90]. The results of Refs. [91,92] are applicable to the TRSM to obtain model predictions for $S, T$ and $U .{ }^{3}$ Flavor constraints are not relevant as the singlets do not change the Yukawa sector. We use HiggsBounds-5.4.0 [98-103] to check for agreement with the bounds from searches for additional Higgs bosons.

Important constraints on the model parameter space arise from the signal rate measurements of the observed $125 \mathrm{GeV}$ Higgs boson, which we denote by $h_{125}$ in the following. These constraints are especially relevant in singlet extensions as there are effectively only two BSM parameters that enter the phenomenology of the $h_{125}$ : its coupling scale factor $\kappa_{125}$ and its decay rate $\mathrm{BR}\left(h_{125} \rightarrow \mathrm{NP}\right)$ into new particles (see Sect. 2.2).

We use HiggsSignals-2.3.0 [104-107] to test for agreement with the observations at the $2 \sigma$ level using a

\footnotetext{
${ }^{3}$ The $W$-boson mass could be used as a single precision observable for models with new particle content, see e.g. Ref. [93] for a discussion within the real singlet extension. We checked the TRSM with an extension of the code presented in Ref. [93] and compared to the updated experimental value $M_{W}=80.379 \pm 0.012 \mathrm{GeV}$ [94-97]. We found no relevant additional constraints from $M_{W}$ in this model.
} 


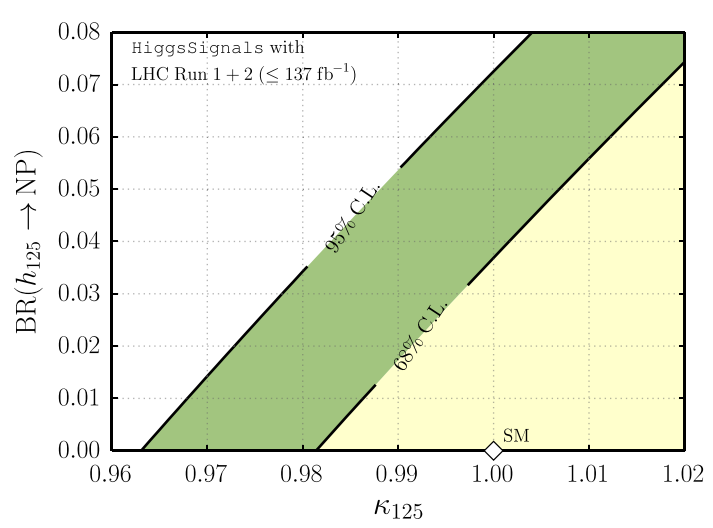

Fig. 2 Constraints from Higgs signal rate measurements on the parameters $\kappa_{125}$ and $\mathrm{BR}\left(h_{125} \rightarrow \mathrm{NP}\right)$ as obtained from HiggsSignals2.3.0

profiled likelihood ratio test with the SM as alternative hypothesis. In practice, the likelihood ratio test statistic is calculated via the difference between the log-likelihoods, which in turn is approximated as $\Delta \chi^{2}=\chi^{2}-\chi_{\mathrm{SM}}^{2}$ within HiggsSignals. As we have two relevant statistical degrees of freedom that can influence the Higgs signal rate predictions (see above), we obtain the $2 \sigma$ confidence region by demanding $\Delta \chi^{2} \leq 6.18$. HiggsSignals-2.3.0 contains the latest measurements from ATLAS [108] and CMS $[10-17,19,20]$ with up to $\sim 137 \mathrm{fb}^{-1}$ of data collected during Run-II at a center-of-mass energy of $13 \mathrm{TeV}$, as well as the ATLAS and CMS combined measurements from Run-I [9].

A further complication may arise in this model in case that two or even all three scalar bosons have a mass around $125 \mathrm{GeV}$. HiggsSignals then automatically takes into account a possible superposition of their signals in the test against the Higgs rate measurements by incoherently summing the contributions of all scalars. This approach neglects any possible interference effects, see Ref. [105] for details. A similar approach is employed in HiggsBounds to combine multiple scalars that lie within the experimental mass resolution.

Assuming only one scalar boson is responsible for the observed signal at $125 \mathrm{GeV}$, we show the constraints from Higgs signal rate measurements in the (simplified) twodimensional parameter plane $\left(\kappa_{125}, \mathrm{BR}\left(h_{125} \rightarrow \mathrm{NP}\right)\right)$ in Fig. $2 .^{4}$ If no BSM decay modes of $h_{125}$ exist, a lower bound on $\kappa_{125}>0.963$ at $95 \%$ C.L. is obtained. For the other limiting case of exactly SM-like couplings, $\kappa_{125}=1$, we find a limit of $\mathrm{BR}\left(h_{125} \rightarrow \mathrm{NP}\right)<7.3 \%$. The $2 \sigma$ limit between these two limiting cases follows approximately a linear slope. The region $\kappa>1$ is only included for completeness in Fig. 2

\footnotetext{
4 The expected sensitivity of Higgs rate measurements at the highluminosity (HL)-LHC in this parameter plane has been presented in Section 6 of Ref. [109].
}

but cannot be realized in the TRSM, see Eq. (18). Note that this analysis is applicable to any model where a singlet scalar mixes with the Higgs boson. This bound can e.g. be applied to Higgs portal models, where it gives a stronger constraint than direct measurements of $\mathrm{BR}\left(h_{125} \rightarrow\right.$ invisible) $[110,111]$.

\subsection{Numerical evaluation}

Based on these constraints we performed a large scan of the TRSM parameter space using an updated private version of the code ScannerS $[35,38,112,113]$. For the determination of viable regions in the parameter space, we apply all of the constraints described above. Note that bounds from signal strength measurements are evaluated with Higgs Signals for each point individually. This guarantees that the possibility that two or even all three Higgs bosons may have masses close to $125 \mathrm{GeV}$ and therefore contribute to the observed signal is correctly accounted for.

We parametrize the model via the input parameters given in Eq. (16). For the numerical results presented in Sect. 4 we independently sample from uniform distributions for each parameter. We allow for the non- $h_{125}$ Higgs masses and the singlet vevs to lie within

$1 \mathrm{GeV} \leq M_{b}, M_{c}, v_{X}, v_{S} \leq 1 \mathrm{TeV}$

and vary the mixing angles throughout their allowed range, Eq. (14). We only keep parameter points that pass all constraints. For the benchmark scenarios in Sect. 5, we fix all parameters apart from the non-SM scalar masses, and scan the two-dimensional parameter space in a grid within the defined parameter ranges.

The singlet vevs are only mildly constrained by current experimental results while theoretical constraints - in particular perturbative unitarity - only require them to not be substantially smaller than the scalar masses. On the other hand, as they enter the triple scalar couplings, Eqs. (19) and (20), they can influence the relative importance of different Higgs-to-Higgs decay modes without changing the remaining phenomenology. We therefore expect that future results from LHC searches for Higgs-to-Higgs decays will be able to constrain the vacuum structure of the singlet fields.

For the SM Higgs production cross sections and decay rates we use the predictions from Refs. [77,114]. The $h_{\mathrm{SM}}$ production cross sections and total width are rescaled according to Eqs. (24) and (25) and combined with leading-order decay widths for the Higgs-to-Higgs decays from Eq. (22). For the $h_{125}$ production rates, we use the results of the $\mathrm{N}^{3} \mathrm{LO}$ calculation in the gluon gluon fusion ( $\mathrm{ggF}$ ) channel [115]. This calculation uses an effective description of the top-induced contributions. For scalar bosons with masses $M_{a} \neq 125 \mathrm{GeV}$ we instead employ results from the NNLO+NNLL calculation [114] that accounts for top-quark 
mass induced effects up to NLO. Indeed, we find that the predictions of these calculations differ sizably for scalar boson masses $M_{a} \gtrsim 2 m_{t}$, for instance, at $M_{a}=400 \mathrm{GeV}$,

$$
\left.\frac{\sigma_{\mathrm{NNLO}+\mathrm{NNLL}}}{\sigma_{\mathrm{N} 3 \mathrm{LO}}}\right|_{M=400 \mathrm{GeV}} \sim 3 .
$$

In the following discussion of collider signatures we assume the production of a single scalar state via the dominant ggF process. In some cases, though, it might be worthwhile to investigate the discovery potential of the subdominant Higgs production processes of vector boson fusion or Higgs-Strahlung, $p p \rightarrow V \phi\left(V=W^{ \pm}, Z\right)$, as these give additional trigger options and may help to reduce the background. We leave the detailed exploration of the prospects for various production modes to future studies.

Scalar pair production can proceed through a top-quark box diagram in addition to single Higgs production followed by a Higgs-to-Higgs decay. For pair production of $h_{125}$ these diagrams and their interference effects with the resonant production are known to be important (see e.g. Refs. [116-119]). For cases other than $h_{125}$-pair production the box diagrams are significantly less important as they are always suppressed by the small $\kappa$ factors of the non- $h_{125}$ scalars. Signal-signal interference effects between different resonant scalars of similar mass have also been shown to significantly impact diHiggs production cross sections [119]. However, such mass configurations play no important role for most of the scenarios in the following discussion.

\section{Implications of current collider searches}

\subsection{Signatures of new scalars decaying to SM particles}

The additional scalar bosons $h_{a}\left(h_{a} \neq h_{125}\right)$ can decay directly to SM particles. The branching ratios of the various SM particle final states $\left(F_{\mathrm{SM}}\right)$ are obtained according to Eq. (28), and their relative rates (i.e. the ratios of branching ratios for different $F_{\mathrm{SM}}$ decay modes) are identical to the corresponding SM predictions for a Higgs boson with mass $M_{a}$. The rate for $h_{a}$ signal processes leading to $F_{\mathrm{SM}}$, normalized to the corresponding SM prediction, can therefore be expressed as

$$
\begin{aligned}
& \frac{\sigma\left(p p \rightarrow h_{a}(+X)\right) \times \mathrm{BR}\left(h_{a} \rightarrow F_{\mathrm{SM}}\right)}{\sigma_{\mathrm{SM}}\left(p p \rightarrow h_{\mathrm{SM}}(+X)\right) \times \mathrm{BR}\left(h_{\mathrm{SM}} \rightarrow F_{\mathrm{SM}}\right)} \\
& =\kappa_{a}^{2} \cdot\left(1-\mathrm{BR}\left(h_{a} \rightarrow \mathrm{NP}\right)\right) .
\end{aligned}
$$

This quantity is shown in Fig. 3 as a function of $M_{a}$ in the low mass region (left panel) and high mass region (right panel) for the sampled parameter points that pass all relevant constraints (see Sect. 3). For $M_{a}$ roughly between 12 and $85 \mathrm{GeV}$ LEP searches for $e^{+} e^{-} \rightarrow h_{a} Z \rightarrow b \bar{b} Z$
[120] lead to an upper limit on the possible signal rate, as shown by the red lines in Fig. 3 (left). At larger mass values $\gtrsim 190 \mathrm{GeV}$, the upper limit originates from LHC searches for $p p \rightarrow h_{a} \rightarrow W^{+} W^{-}$and ZZ. The latest ATLAS [121] and CMS [122] limits are overlaid as green and orange lines, respectively, in Fig. 3 (right). For very large mass values $\gtrsim 700 \mathrm{GeV}$ direct LHC searches are not yet sensitive to probe the parameter space. In addition, we include in Fig. 3 the upper limit inferred indirectly via the sum rule, Eq. (18), from the rate measurements of the $125 \mathrm{GeV}$ Higgs state. These lead to an upper limit of $\kappa_{a}^{2} \leq 7.3 \%$ (see Sect. 3.2), except in the mass region around $125 \mathrm{GeV}$ where $h_{a}$ potentially contributes to the observed Higgs signal.

\subsection{Signatures of resonant scalar pair production}

The model allows for resonant scalar pair-production at the LHC, or, in other words, the direct production of a single scalar $h_{a}$ followed by the "symmetric" or "asymmetric" decay into identical or different scalar states, respectively. Specifically,

$p p \rightarrow h_{a}(+X) \rightarrow h_{b} h_{b}(+X)$,

$p p \rightarrow h_{3}(+X) \rightarrow h_{1} h_{2}(+X)$,

where, in the symmetric case, Eq. (38), $a=2, b=1$ or $a=3, b \in\{1,2\}$, and $X$ denotes not further defined objects that may be produced in association with the scalar state (e.g., jets, vector bosons, etc.). The $h_{125}$ can be either of the three scalar states $h_{a}(a \in\{1,2,3\})$.

Processes of the symmetric type, Eq. (38), leading to pair production of $h_{125}$ are already being investigated, see e.g. Refs. [58-69] for recent LHC Run-II searches. Figure 4 (left) shows the $13 \mathrm{TeV}$ LHC signal rate for the resonant scalar pair production process $p p \rightarrow h_{a} \rightarrow h_{125} h_{125}$ ( $a \in\{2,3\})$ as a function of the $h_{a}$ mass, $M_{a}$. Figure 4 contains the complete sample of allowed parameter points generated according to Sect. 3.3. Overlaid are the most recent experimental limits on this process from the ATLAS [69] and CMS [66] collaborations. Figure 4 (left) illustrates that experimental searches in this channel are beginning to directly constrain the TRSM for resonance masses between around $380 \mathrm{GeV}$ and $550 \mathrm{GeV}$. In contrast, LHC searches [70-76] for the inverted signature of single-production of $h_{125}$ which then decays into a pair of light $h_{a}(a \in\{1,2\})$ are not yet sensitive, as the indirect constraints from Higgs signal rates on the possible new decay modes, $\mathrm{BR}\left(h_{125} \rightarrow \mathrm{NP}\right) \leq 7.3 \%$ (see Fig. 2), are much stronger than the direct limits from these searches. ${ }^{5}$ Both of these processes are under active

\footnotetext{
5 Currently, the strongest limit from $h_{125} \rightarrow h_{a} h_{a}$ searches is obtained in the $b \bar{b} \tau^{+} \tau^{-}$final state [71] at $M_{a} \simeq 35 \mathrm{GeV}$, amounting to around $\operatorname{BR}\left(h_{125} \rightarrow h_{a} h_{a}\right) \leq 25 \%$ (assuming $h_{a}$ to decay exclusively to SM particles) in the TRSM.
} 


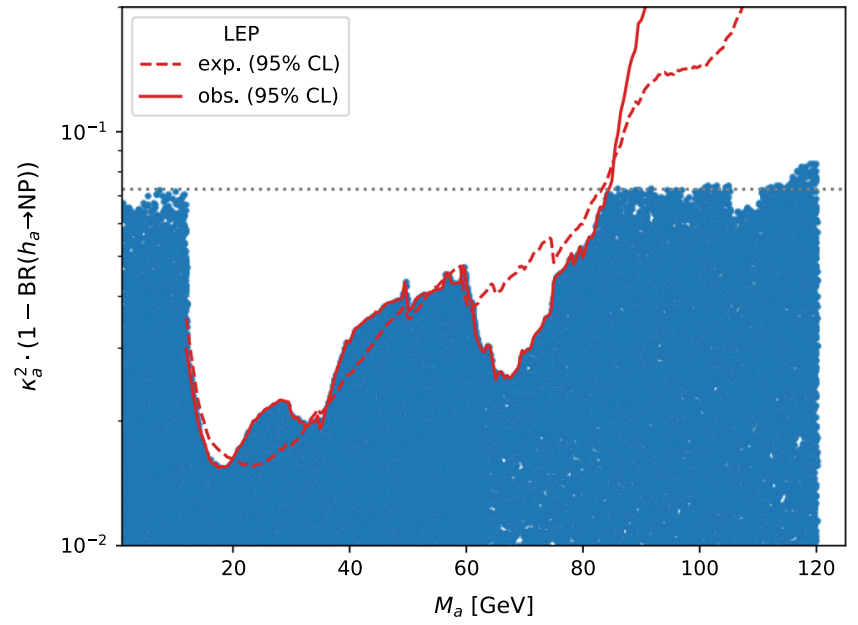

Fig. 3 SM-normalized signal rate for additional Higgs bosons decaying to SM particle final states as a function of its mass, $M_{a}$, for all parameter points passing all relevant constraints (blue points). In the low mass region (left panel) we include the observed (solid line) and expected (dashed line) limit from LEP searches in the $e e \rightarrow h_{a} Z \rightarrow$

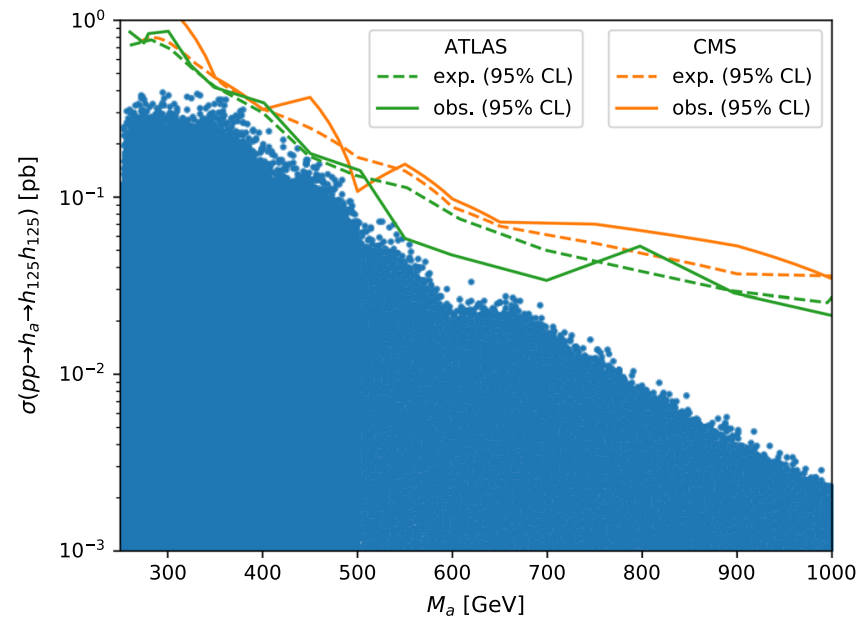

Fig. 4 Left panel: signal rate for the process $p p \rightarrow h_{a} \rightarrow h_{125} h_{125}$ at the $13 \mathrm{TeV}$ LHC as a function of the $h_{a}$ mass, $M_{a}$, for all parameter points passing all relevant constraints (blue points). The current expected and observed upper limits on this process from ATLAS [69]

experimental investigation and we expect the bounds to improve in the future.

We will now turn to the more exotic signatures resulting from Eqs. (38) and (39) that are not yet under active investigation. Following the processes in Eqs. (38) and (39), the two produced scalar states may further decay directly to SM particles. Alternatively, an $h_{2}$ final state may decay into the two lightest scalars: $h_{2} \rightarrow h_{1} h_{1}$. This can lead to interesting decay cascades leading to three or four scalar states that eventually decay to SM particles. The possible decay patterns within our model are depicted in a generic form in

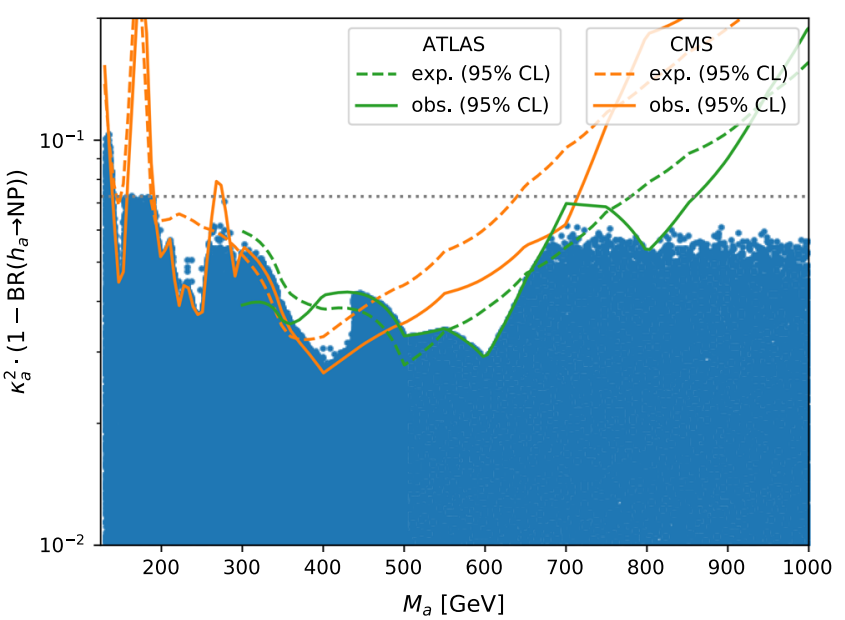

$b b Z$ channel [120]. In the high mass region (right panel) the ATLAS [121] and CMS [122] observed and expected limits from the latest $p p \rightarrow h_{a} \rightarrow Z Z / W W$ searches are displayed. The dotted gray line indicates the indirect limit on $\kappa^{2}$ from Higgs rate measurements

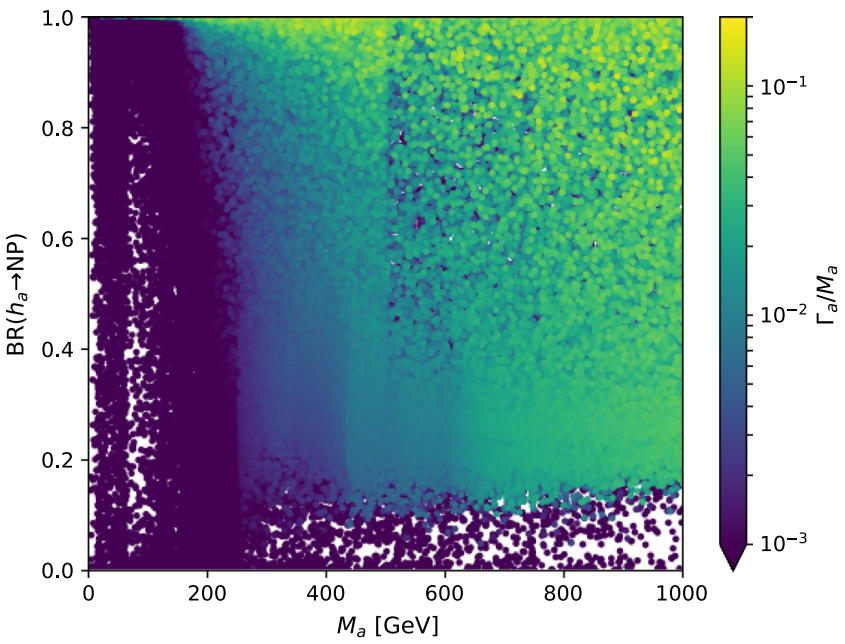

(green lines) and CMS [66] (orange lines) are overlaid. Right panel: total decay width over mass, $\Gamma_{a} / M_{a}$, of the resonant scalar as a function of $M_{a}$ and the decay rate $\mathrm{BR}\left(h_{a} \rightarrow \mathrm{NP}\right)$. Parameter points with larger $\Gamma_{a} / M_{a}$ values are plotted on top of points with smaller values

Fig. 5. Here and in the following we denote final states from Higgs decays composed of SM particles (i.e. gauge bosons or fermions) generically $F_{\mathrm{SM}}$, unless otherwise specified. For the more complicated final states we will use $F_{\mathrm{SM}}^{n}$ to denote an $n$-particle SM final state, where we count the SM particles before their decay (i.e., $W^{ \pm}, Z$, and $t$ are counted as one particle). As discussed above, the relative fractions of their decay rates solely depend on the mass of the decaying Higgs state.

We find that all possible Higgs-to-Higgs decay signatures, Eqs. (38) and (39), can appear at sizable rates in the 


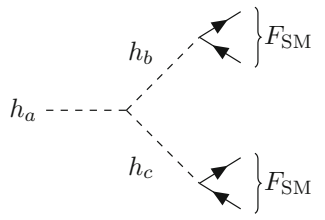

(a) direct

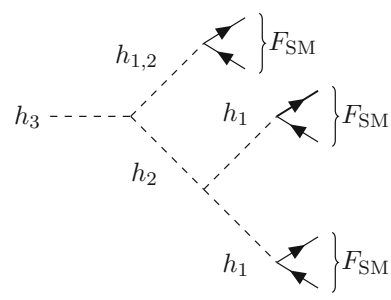

(b) single cascade

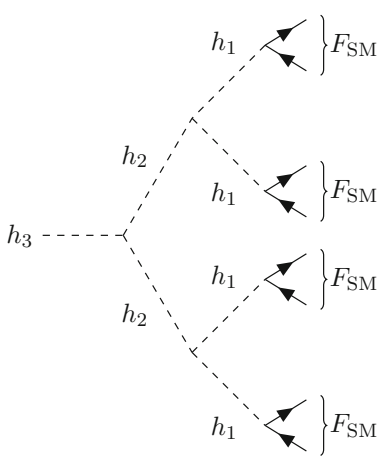

(c) double cascade
Fig. 5 Possible Higgs-to-Higgs decay signatures involving three neutral (mass ordered) scalars $h_{a}(a \in\{1,2,3\}): \mathbf{a} h_{a} \rightarrow h_{b} h_{c}$ (with $a>b, c)$ with successive decay of $h_{b}$ and $h_{c}$ to SM particles; b $h_{3} \rightarrow h_{2} h_{c}$ (with $c \in\{1,2\}$ ) with successive decay $h_{2} \rightarrow h_{1} h_{1}$ and $h_{k}$ as well as all $h_{1}$ decaying to SM particles; $\mathbf{c} h_{3} \rightarrow h_{2} h_{2} \rightarrow h_{1} h_{1} h_{1} h_{1}$ and all $h_{1}$ decaying to SM particles

allowed TRSM parameter space. In the next section we therefore design six two-dimensional benchmark scenarios that highlight these signatures in detail, and are tailored to initiate dedicated experimental studies and facilitate the design of corresponding searches. As a final remark, we briefly want to comment on the possible size of the total width of the resonantly-produced scalar state $h_{a}$. Figure 4 (right) shows the ratio of the total width over the mass, $\Gamma_{a} / M_{a}$, in dependence of $M_{a}$ and the sum of $h_{a}$ decays to scalar states, $\mathrm{BR}\left(h_{a} \rightarrow \mathrm{NP}\right)$. Parameter points with larger values of $\Gamma_{a} / M_{a}$ overlay parameter points with smaller values. We can clearly see that parameter points with larger $\Gamma_{a} / M_{a}$ tend to have sizable decay rates to scalar states. However, overall, $\Gamma_{a} / M_{a}$ never exceeds values greater than around $18 \%$ in the considered mass range up to $1 \mathrm{TeV}$. In the discussion of the benchmark scenarios below we will comment on cases where $\Gamma_{a} / M_{a} \gtrsim 1 \%$.

\section{Benchmark scenarios}

In this section we define six benchmark scenarios in order to motivate and enable dedicated experimental studies of Higgsto-Higgs decay signatures. Each scenario focusses on one (or more) novel signatures and features a (close-to) maximal signal yield that can be expected within the model while obeying the constraints described in Sect. 3. The benchmark scenarios are defined as two-dimensional planes where all model parameters except for the two non- $h_{125}$ scalar masses are fixed. A brief overview of the benchmark scenarios is given in Table 1. For each benchmark scenario,
BP1-BP6, it specifies the Higgs state $h_{a}$ that is identified with the observed Higgs state, $h_{125}$, the target Higgs-toHiggs decay signature, as well as the possibilities of phenomenologically relevant ${ }^{6}$ successive Higgs decays, potentially leading to single or double cascade decay signatures (see Fig. 5).

The model parameters for all scenarios as well as the coupling scale factors $\kappa_{a}$ are given in Table 2. All cross section values given in the following refer to production of the initial scalar through ggF at the $13 \mathrm{TeV}$ LHC.

We employ a factorized approach relying on the narrow width approximation. For each benchmark scenario we will show both the $\mathrm{BR}\left(h_{a} \rightarrow h_{b} h_{c}\right)(a, b, c \in\{1,2,3\}, a \neq b, c)$ and the cross section

$$
\begin{aligned}
& \sigma\left(p p \rightarrow h_{a} \rightarrow h_{b} h_{c}\right) \\
& \quad=\left.\kappa_{a}^{2} \sigma\left(g g \rightarrow h_{\mathrm{SM}}\right)\right|_{M_{a}} \cdot \operatorname{BR}\left(h_{a} \rightarrow h_{b} h_{c}\right) .
\end{aligned}
$$

In all scenarios where either $b=c$ or $h_{b, c} \equiv h_{125}$ there is only one unknown BSM mass in the final state $h_{b} h_{c}$. In this case we will employ a further factorization where we present the $\mathrm{BR}\left(h_{b} h_{c} \rightarrow F_{\mathrm{SM}}\right)$ as a function of the remaining mass parameter. In this case the full cross section into a given SM final state can be obtained by

$$
\begin{aligned}
& \sigma\left(p p \rightarrow h_{a} \rightarrow h_{b} h_{c} \rightarrow F_{\mathrm{SM}}\right) \\
& \quad=\sigma\left(p p \rightarrow h_{a} \rightarrow h_{b} h_{c}\right) \cdot \operatorname{BR}\left(h_{b} h_{c} \rightarrow F_{\mathrm{SM}}\right),
\end{aligned}
$$

where potential cascades, Fig. 5 , are included in the $\mathrm{BR}\left(h_{b} h_{c}\right.$ $\left.\rightarrow F_{\mathrm{SM}}\right)$ for $F_{\mathrm{SM}}^{6}$ and $F_{\mathrm{SM}}^{8}$.

All of the benchmark scenarios presented in the following are exemplary for the corresponding signature within the TRSM. There are always alternative choices for the fixed parameters that may lead to different cross sections, branching ratios, or regions excluded by some constraints. As such, the regions of parameter space that are excluded by some constraint in a benchmark scenario should under no circumstances discourage experimental searches in this parameter region.

\subsection{BP1: $h_{125} \rightarrow h_{1} h_{2}$}

In the first benchmark scenario, BP1, we identify the heaviest scalar state $h_{3}$ with $h_{125}$, and focus on the asymmetric decay $h_{125} \rightarrow h_{1} h_{2}$. The parameter values (see Table 2 ) are chosen such that the couplings of $h_{3}$ to SM particles are nearly identical to the $\mathrm{SM}$ predictions, $\kappa_{3} \simeq 1$. At the same time,

\footnotetext{
${ }^{6}$ For instance, in BP2, the successive decay $h_{2} \rightarrow h_{1} h_{1}$ could in principle occur for the case that $M_{1}<62.5 \mathrm{GeV}$, however, Higgs signal rate measurements strongly constrain the possible decay rate, and we do not further consider this possiblity here.
} 
Table 1 Overview of the benchmark scenarios: The second column denotes the Higgs mass eigenstate that we identify with the observed Higgs boson, $h_{125}$, the third column names the targeted decay mode of the resonantly produced Higgs state, and the fourth column lists possible relevant successive decays of the resulting Higgs states

Table 2 Input parameter values and coupling scale factors, $\kappa_{a}$ $(a=1,2,3)$, for the six defined benchmark scenarios. The doublet vev is set to $v=246 \mathrm{GeV}$ for all scenarios

\begin{tabular}{llll}
\hline Benchmark scenario & $h_{125}$ candidate & Target signature & Possible successive decays \\
\hline BP1 & $h_{3}$ & $h_{125} \rightarrow h_{1} h_{2}$ & $h_{2} \rightarrow h_{1} h_{1}$ if $M_{2}>2 M_{1}$ \\
BP2 & $h_{2}$ & $h_{3} \rightarrow h_{1} h_{125}$ & - \\
BP3 & $h_{1}$ & $h_{3} \rightarrow h_{125} h_{2}$ & $h_{2} \rightarrow h_{125} h_{125}$ if $M_{2}>250 \mathrm{GeV}$ \\
BP4 & $h_{3}$ & $h_{2} \rightarrow h_{1} h_{1}$ & - \\
BP5 & $h_{2}$ & $h_{3} \rightarrow h_{1} h_{1}$ & - \\
BP6 & $h_{1}$ & $h_{3} \rightarrow h_{2} h_{2}$ & $h_{2} \rightarrow h_{125} h_{125}$ if $M_{2}>250 \mathrm{GeV}$ \\
\hline
\end{tabular}

\begin{tabular}{lllllll}
\hline Parameter & \multicolumn{6}{l}{ Benchmark scenario } \\
\cline { 2 - 6 } & BP1 & BP2 & BP3 & BP4 & BP5 & BP6 \\
\hline$M_{1}(\mathrm{GeV})$ & {$[1,62]$} & {$[1,124]$} & 125.09 & {$[1,62]$} & {$[1,124]$} & 125.09 \\
$M_{2}(\mathrm{GeV})$ & {$[1,124]$} & 125.09 & {$[126,500]$} & {$[1,124]$} & 125.09 & {$[126,500]$} \\
$M_{3}(\mathrm{GeV})$ & 125.09 & {$[126,500]$} & {$[255,650]$} & 125.09 & {$[126,500]$} & {$[255,1000]$} \\
$\theta_{h s}$ & 1.435 & 1.352 & -0.129 & -1.284 & -1.498 & 0.207 \\
$\theta_{h x}$ & -0.908 & 1.175 & 0.226 & 1.309 & 0.251 & 0.146 \\
$\theta_{s x}$ & -1.456 & -0.407 & -0.899 & -1.519 & 0.271 & 0.782 \\
$v_{s}(\mathrm{GeV})$ & 630 & 120 & 140 & 990 & 50 & 220 \\
$v_{x}(\mathrm{GeV})$ & 700 & 890 & 100 & 310 & 720 & 150 \\
$\kappa_{1}$ & 0.083 & 0.084 & 0.966 & 0.073 & 0.070 & 0.968 \\
$\kappa_{2}$ & 0.007 & 0.976 & 0.094 & 0.223 & -0.966 & 0.045 \\
$\kappa_{3}$ & -0.997 & -0.203 & 0.239 & 0.972 & -0.250 & 0.246 \\
\hline
\end{tabular}

the parameter choice maximizes - within the experimentally allowed range - the branching ratio $\mathrm{BR}\left(h_{125} \rightarrow h_{1} h_{2}\right)$, which is shown in Fig. 6 (top left) as a function of $M_{1}$ and $M_{2}$. In Fig. 6 (top right) we show the corresponding signal rate for inclusive production via gluon gluon fusion. We find that the BR for $h_{3} \rightarrow h_{1} h_{2}$ reaches up to $7-8 \%$ which translates into a signal rate of around $3 \mathrm{pb}$. These maximal branching ratios are reached in the intermediate mass range for $h_{2}, M_{2} \sim 60-80 \mathrm{GeV}$. This feature is caused by the fact that the triple Higgs couplings are proportional to the masses (see Eq. (20)). Therefore, although phase space opens up significantly for light decay products, the branching ratios become smaller for $M_{2}<40 \mathrm{GeV}$. In the hatched region in Fig. 6 the decay rate slightly exceeds the $2 \sigma$ upper limit inferred from the LHC Higgs rate measurements (using HiggsSignals). We stress again that this excluded area is dependent on our parameter choices and strongly encourage experimental searches to cover the whole mass range.

Due to the sum rule, Eq. (18), the coupling scale factors $\kappa_{1,2}$ have to be very close to zero in order to achieve $\kappa_{3} \sim 1$. This means that the couplings of $h_{1}$ and $h_{2}$ to SM particles are strongly suppressed. As a result, if the decay channel $h_{2} \rightarrow$ $h_{1} h_{1}$ is kinematically open, $M_{2}>2 M_{1}$, it is the dominant decay mode leading to a significant rate for the $h_{1} h_{1} h_{1}$ final state. In BP1 we find that $\operatorname{BR}\left(h_{2} \rightarrow h_{1} h_{1}\right) \simeq 100 \%$ in this kinematic regime (i.e. above the red line in Fig. 6) with a very sharp transition at the threshold. If in addition $M_{1} \gtrsim 10 \mathrm{GeV}$ the $h_{1}$ decays dominantly into $b \bar{b}$ leading to a sizeable rate for the $b \bar{b} b \bar{b} b \bar{b}$ final state as shown in Fig. 6 (bottom right).

If the $h_{2} \rightarrow h_{1} h_{1}$ decay is kinematically closed, $M_{2}<$ $2 M_{1}$, both scalars $h_{1}$ and $h_{2}$ decay directly to SM particles, with BRs identical to a SM-like Higgs boson with the corresponding mass (see Fig. 1). Therefore, for masses $M_{1}, M_{2} \gtrsim 10 \mathrm{GeV}$, the $b \bar{b} b \bar{b}$ final state dominates, as shown in Fig. 6 (bottom left), while at smaller masses, combinations with $\tau$-leptons and eventually final states containing charm quarks and muons become relevant.

\section{$5.2 \mathrm{BP} 2: h_{3} \rightarrow h_{1} h_{125}$}

In the second benchmark scenario, BP2, we identify $h_{125} \equiv$ $h_{2}$ and consider the production of $h_{3}$ followed by the asymmetric decay $h_{3} \rightarrow h_{1} h_{125}$. The scenario is defined in the $\left(M_{1}, M_{3}\right)$ parameter plane, and the remaining parameters are fixed to the values given in Table 2 . The mixing angles are chosen such that the production rate of $h_{3}$ is maximized, while the $h_{2}$ properties remain consistent with the measured Higgs signal rates. This results in a $h_{3}$ production rate of roughly $4 \%$ of the production cross section for a $h_{\mathrm{SM}}$ at the same mass. 

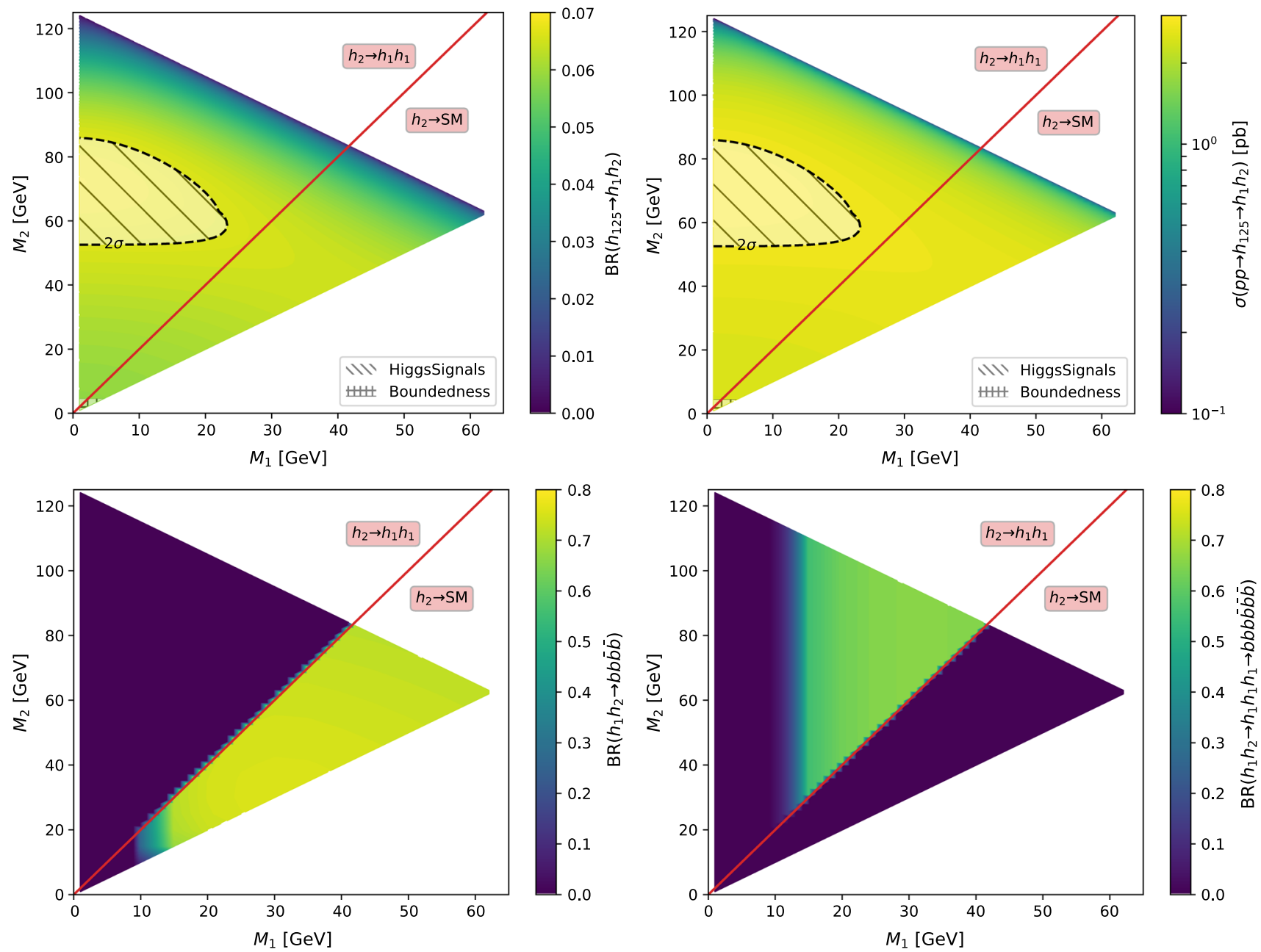

Fig. 6 Benchmark plane BP1 for the decay signature $h_{125} \rightarrow h_{1} h_{2}$ with $h_{125} \equiv h_{3}$, defined in the $\left(M_{1}, M_{2}\right)$ plane. The color code shows $\mathrm{BR}\left(h_{3} \rightarrow h_{1} h_{2}\right)$ (top left panel) and the $13 \mathrm{TeV}$ LHC signal rate for $p p \rightarrow h_{3} \rightarrow h_{1} h_{2}$ (top right panel). The red line separates the

region $M_{2}>2 M_{1}$, where $\mathrm{BR}\left(h_{2} \rightarrow h_{1} h_{1}\right) \approx 100 \%$, from the region $M_{2}<2 M_{1}$, where $\mathrm{BR}\left(h_{2} \rightarrow F_{\mathrm{SM}}\right) \approx 100 \%$. The BR of the $h_{1} h_{2}$ state into $b \bar{b} b \bar{b}$ and - through a $h_{2} \rightarrow h_{1} h_{1}$ cascade $-b \bar{b} b \bar{b} b \bar{b}$ final states are shown in the bottom left and right panels, respectively
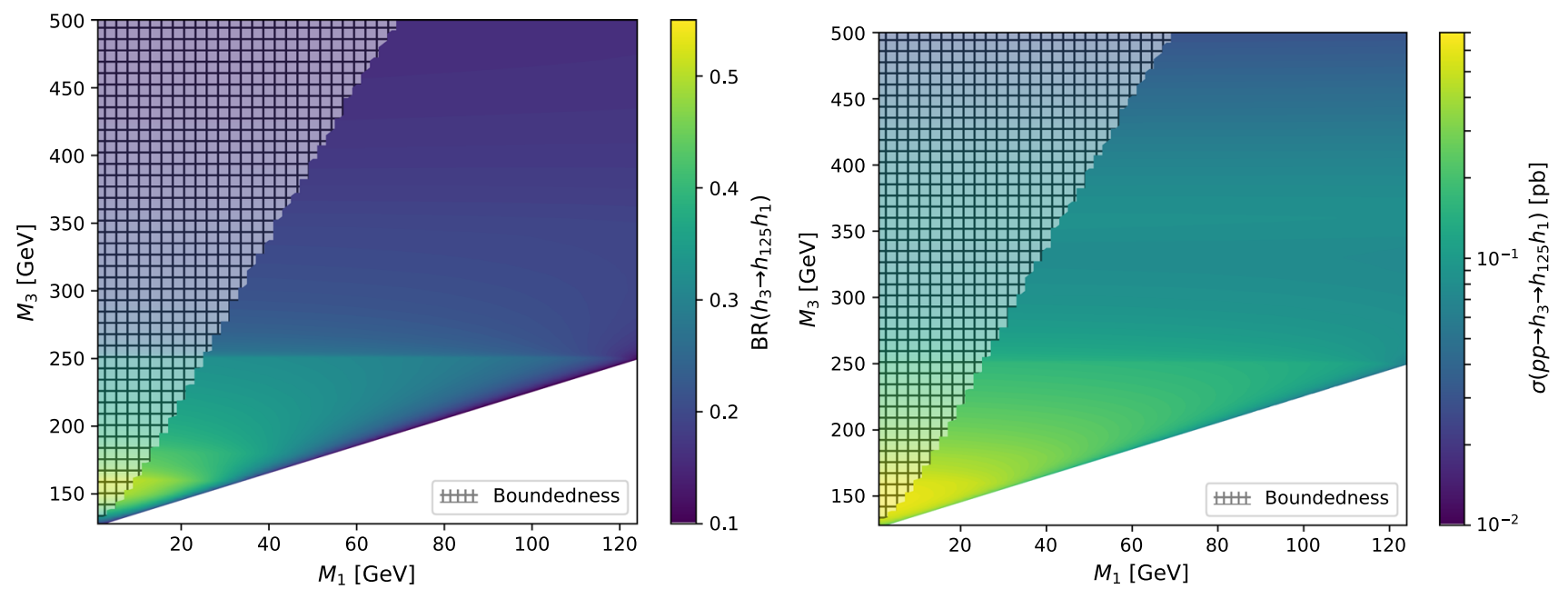

Fig. 7 Benchmark plane BP2 for the decay signature $h_{3} \rightarrow h_{1} h_{125}$ with $h_{125} \equiv h_{2}$, defined in the $\left(M_{1}, M_{3}\right)$ plane. The color code shows $\mathrm{BR}\left(h_{3} \rightarrow h_{1} h_{2}\right)$ (left panel) and the signal rate for $p p \rightarrow h_{3} \rightarrow h_{1} h_{2}$ (right panel) 


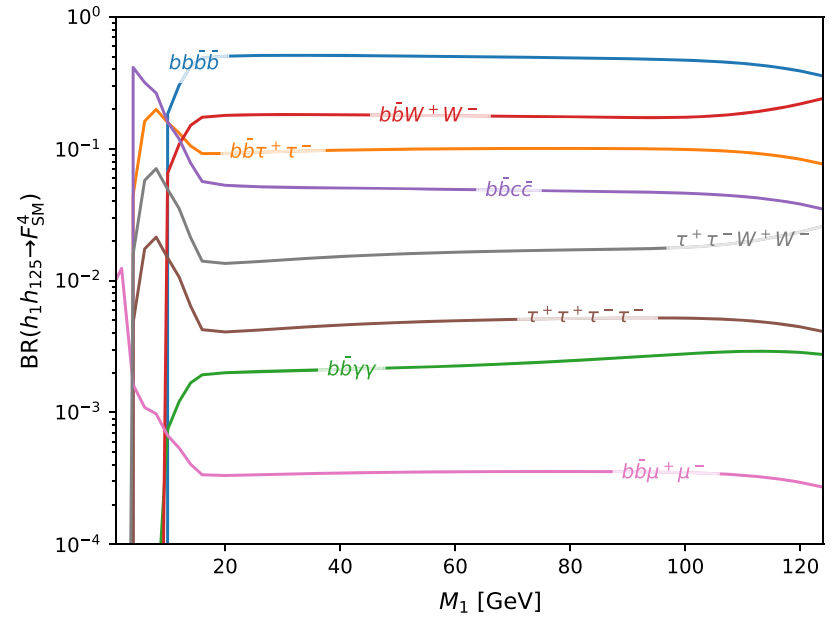

Fig. 8 Branching ratios of the $h_{1} h_{125}$ state decaying into selected SM final states as a function of $M_{1}$ for $\mathbf{B P 2}$

The phenomenology of $\mathbf{B P 2}$ is illustrated by Fig. 7. The $\operatorname{BR}\left(h_{3} \rightarrow h_{1} h_{2}\right)$ shown in Fig. 7 (left) mostly stays above $20 \%$ for $M_{3} \lesssim 350 \mathrm{GeV}$, reaching maximal values of around $50-55 \%$ in the low mass region, $M_{3} \sim$ $150-170 \mathrm{GeV}$. In this region, the corresponding signal rate in Fig. 7 (right) is about $0.6 \mathrm{pb}$. It remains above $50 \mathrm{fb}$ as long as $M_{3} \lesssim 450 \mathrm{GeV}$. The shaded region in Fig. 7 is excluded by boundedness of the scalar potential. Again, this constraint depends strongly on the values of the model parameters and should not discourage experimental efforts to perform modelindependent searches in this mass range. The total width of $h_{3}$ can reach maximal values of $\Gamma_{3} / M_{3} \sim 1.1 \%$ in this benchmark scenario for $M_{3} \gtrsim 480 \mathrm{GeV}$.

The branching ratios for decays to SM final states originating from the $h_{1} h_{125}$ two-particle state are shown in Fig. 8 for BP2 as a function of $M_{1}$. In most of the mass range, the $b \bar{b} b \bar{b}$ final state dominates, followed by $b \bar{b} W^{+} W^{-}$and $b \bar{b} \tau^{+} \tau^{-}$final states.

The cascade decay $h_{125} \equiv h_{2} \rightarrow h_{1} h_{1}$ is in principle possible if kinematically allowed and in compliance with the observed $h_{125}$ properties. However, we chose $\kappa_{2}^{2}$ small in order to maximize $\kappa_{3}$ within the experimental constraints. From Fig. 2 we see that, at the corresponding value of $\kappa_{2}$, $\mathrm{BR}\left(h_{125} \rightarrow h_{1} h_{1}\right)$ must not exceed $\sim 2.5 \%$. In BP2 this decay rate is always below $0.1 \%$.

Besides the asymmetric decay $h_{3} \rightarrow h_{1} h_{2}$ the symmetric decays $h_{3} \rightarrow h_{1} h_{1}$ and $h_{3} \rightarrow h_{2} h_{2}$ are also present in this scenario. The decay $h_{3} \rightarrow h_{1} h_{1}$ has a rate $\gtrsim 25 \%$ in the mass range $M_{3} \lesssim 250 \mathrm{GeV}$. The decay mode $h_{3} \rightarrow h_{2} h_{2}$ only becomes kinematically open for $M_{3} \gtrsim 2 M_{2}=250 \mathrm{GeV}$, and reaches rates up to $\sim 28 \%$. Although these rates are not negligible in BP2, we shall define dedicated benchmark scenarios BP5 and BP6 below where these decay modes clearly dominate.

\subsection{BP3: $h_{3} \rightarrow h_{125} h_{2}$}

In benchmark scenario BP3 we identify $h_{125} \equiv h_{1}$ and consider the production of $h_{3}$ followed by the asymmetric decay $h_{3} \rightarrow h_{125} h_{2}$. Similar to the $\mathbf{B P 2}$ scenario the mixing angles are chosen to maximize $\kappa_{3}^{2} \simeq 5.7 \%$ and $\mathrm{BR}\left(h_{3} \rightarrow h_{1} h_{2}\right)$. The benchmark plane corresponding to the parameters given in Table 2 is shown in Fig. 9.

The $\operatorname{BR}\left(h_{3} \rightarrow h_{125} h_{2}\right)$ shown in Fig. 9 (left) is $\gtrsim 35 \%$ throughout the benchmark plane except for the region very close to threshold. It reaches values around $50 \%$ in the parameter region $M_{3} \lesssim 2 M_{2}$. The signal cross section, $\sigma\left(p p \rightarrow h_{3} \rightarrow h_{1} h_{2}\right)$ shown in Fig. 9 (right), reaches up to $0.3 \mathrm{pb}$ while $M_{3} \lesssim 500 \mathrm{GeV}$. At large values of $M_{3} \gtrsim$ $500-600 \mathrm{GeV}$ the parameter space is partly constrained by perturbative unitarity, and if simultaneously $M_{1} \lesssim 150 \mathrm{GeV}$ the potential can become unbounded from below, as indicated by the shaded regions. Very close to its kinematic threshold, $M_{3} \simeq M_{1}+125 \mathrm{GeV}$, the decay $h_{3} \rightarrow h_{125} h_{1}$ is strongly suppressed. In this case, constraints can be derived from current LHC searches for heavy resonances, in particular for the process $p p \rightarrow h_{3} \rightarrow Z Z[121,122]$. The total width of $h_{3}$ is maximal for the largest allowed values of $M_{3}$ and reaches $\Gamma_{3} / M_{3} \sim 4 \%$ for $M_{3} \gtrsim 600 \mathrm{GeV}$.

If $M_{2}<250 \mathrm{GeV}$ BSM decay modes of $h_{2}$ are prohibited and its decay rates are identical to an $h_{\mathrm{SM}}$ of the same mass (see Fig. 1). In this region the $h_{125} h_{2}$ state dominantly decays into final states involving $b$-quarks and heavy gauge bosons as shown in Fig. 10. As soon as $M_{2}>250 \mathrm{GeV}$ the decay $h_{2} \rightarrow h_{125} h_{125}$ becomes dominant, quickly reaching a rate of $\sim 70 \%$. Above threshold this rate remains largely independent of $M_{2}$. The decay BRs of the resulting $h_{125} h_{125} h_{125}$ state to the most important six particle SM final states, $F_{\mathrm{SM}}^{6}$, are given in Table 3 . The first row lists the direct branching ratios of $h_{125} h_{125} h_{125}$ while the second row includes the factor $\operatorname{BR}\left(h_{2} \rightarrow h_{125} h_{125}\right) \approx 68 \%$, which is an approximation obtained in the mass region $260 \mathrm{GeV}<$ $M_{2}<500 \mathrm{GeV}$. The resulting values can thus be compared directly to the BRs of the four particle $F_{\mathrm{SM}}^{4}$ in Fig. 10. For instance, we find that rates for $b \bar{b} b \bar{b} b \bar{b}, b \bar{b} b \bar{b} W^{+} W^{-}$and $b \bar{b} W^{+} W^{-}$final states are of comparable size for $M_{2} \gtrsim$ $270 \mathrm{GeV}$.

The maximal production rates for the $h_{3} \rightarrow h_{1} h_{2} \rightarrow F_{\mathrm{SM}}^{4}$ and $h_{3} \rightarrow h_{1} h_{2} \rightarrow h_{1} h_{1} h_{1} \rightarrow F_{\mathrm{SM}}^{6}$ signatures amount to around $0.3 \mathrm{pb}$ and $0.14 \mathrm{pb}$, respectively, where the latter is found when both decays are just above threshold, $M_{3} \simeq$ $380 \mathrm{GeV}$ and $M_{2} \simeq 255 \mathrm{GeV}$.

In BP3, the competing symmetric decay $h_{3} \rightarrow h_{2} h_{2}$ reaches rates of $\simeq 20 \%$ if kinematically allowed. Otherwise the decay $h_{3} \rightarrow h_{125} h_{125}$ reaches similar values (and becomes dominant in the threshold region, $M_{3} \sim M_{1}$ $+M_{2}$ ). 


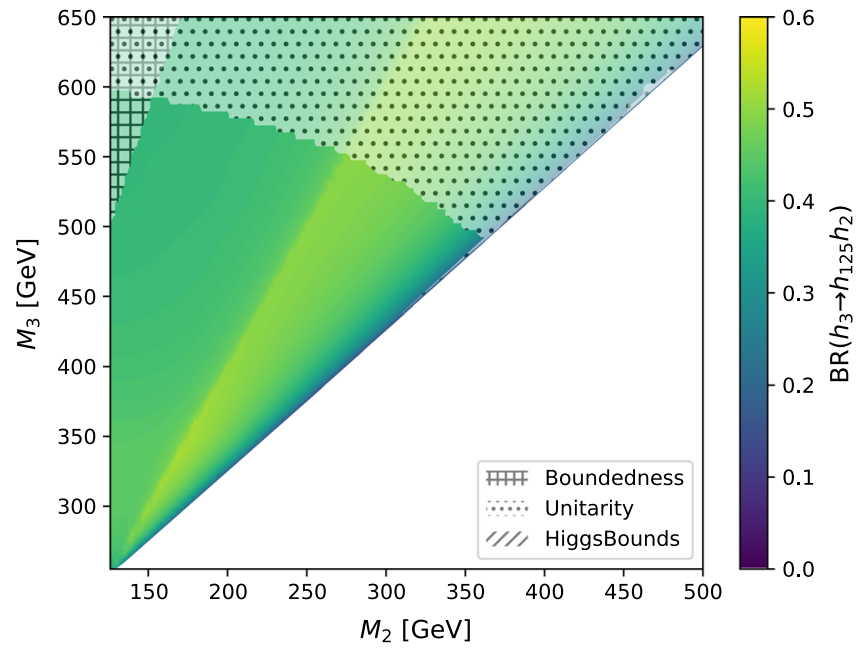

Fig. 9 Benchmark plane BP3 for the decay signature $h_{3} \rightarrow h_{125} h_{2}$ with $h_{125} \equiv h_{1}$, defined in the $\left(M_{2}, M_{3}\right)$ plane. The color code shows $\mathrm{BR}\left(h_{3} \rightarrow h_{125} h_{2}\right)$ (left panel) and the signal rate for $p p \rightarrow h_{3} \rightarrow$

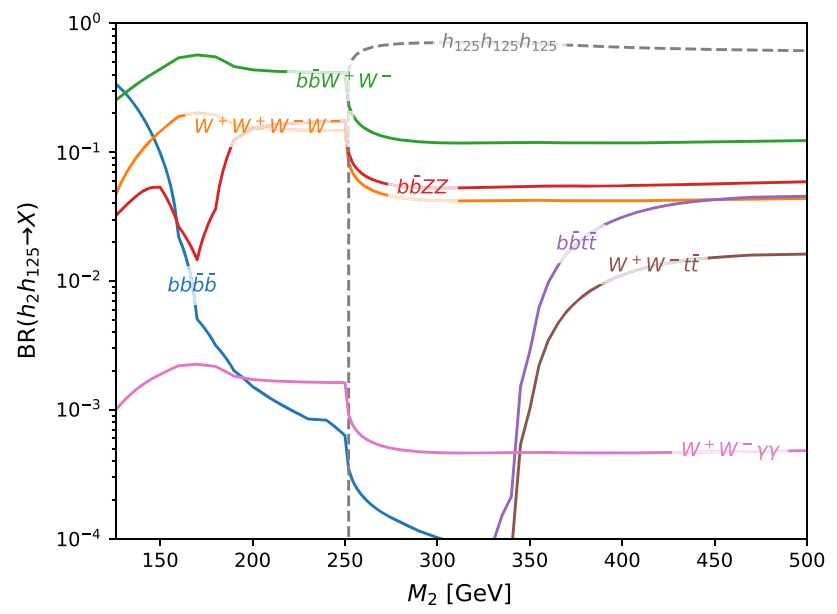

Fig. 10 Branching ratios of the $h_{125} h_{2}$ state as a function of $M_{2}$ for BP3. Included are a selection of decay modes into SM particles as well as the cascade decay to $h_{125} h_{125} h_{125}$

\subsection{BP4: $h_{2} \rightarrow h_{1} h_{1}$ with $h_{125} \equiv h_{3}$}

We now turn to the symmetric Higgs-to-Higgs decay signatures. In benchmark scenario BP4 we identify $h_{125} \equiv h_{3}$ and focus on the production of $h_{2}$ followed by its decay $h_{2} \rightarrow h_{1} h_{1}$. In order to avoid constraints from the Higgs

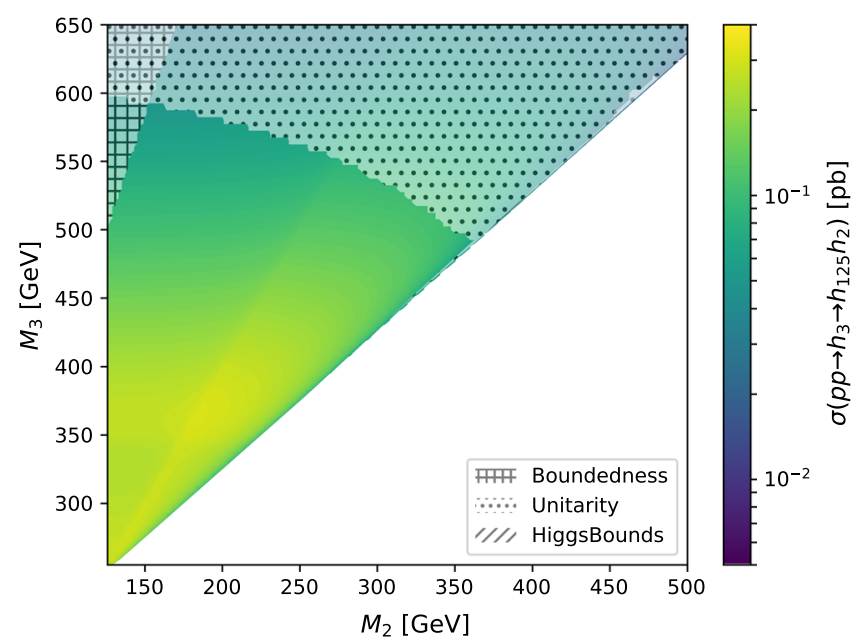

$h_{125} h_{2}$ (right panel). The shaded regions are excluded by boundedness from below, perturbative unitarity, and searches for heavy scalar resonances in diboson final states $[121,122]$

rate measurements on the possible decays $h_{125} \rightarrow h_{a} h_{b}$ ( $a, b \in\{1,2\})$, the relevant couplings must be tuned to rather small values while keeping $\left|\kappa_{2}\right|$ relatively large to ensure sizeable direct production of $h_{2}$. The parameter choices for BP4 are listed in Table 2.

Figure 11 shows the collider phenomenology of BP4. The branching ratio $\mathrm{BR}\left(h_{2} \rightarrow h_{1} h_{1}\right)$ is larger than $50 \%$ throughout the allowed parameter plane, as shown in Fig. 11 (left). For $M_{2} \gtrsim 40 \mathrm{GeV}$ it is by far the dominant decay mode of $h_{2}$ with a BR of more than $90 \%$. As the produced scalar boson is light, the signal rates shown in Fig. 11 (right) are enhanced by the large $\mathrm{ggF}$ cross section for light scalars. Even though $h_{2}$ is only produced with a rate of about $\kappa_{2}^{2} \sim 5 \%$ of the SM Higgs cross section at the same mass, we still obtain signal rates of $\mathcal{O}(100 \mathrm{pb})$ in the low mass region $M_{2} \lesssim 20 \mathrm{GeV}$. However, this parameter region is partly constrained by LEP searches for $e^{+} e^{-} \rightarrow Z h_{2} \rightarrow Z(b \bar{b})$ [120]. For $M_{2} \geq 20 \mathrm{GeV}$, where this limit is no longer sensitive, the signal rate can still reach $60 \mathrm{pb}$. Still, the signature remains experimentally challenging as the decay products for these low $M_{1}$ will be very soft.

The BRs for the decay modes of the $h_{1} h_{1}$ state into SM particles are shown in Fig. 12. For $M_{1} \gtrsim 10 \mathrm{GeV}$ the decay

Table 3 Decay rates of the $h_{125} h_{125} h_{125}$ state in BP3, leading to a six-particle SM final state, $F_{\mathrm{SM}}^{6}$. The second row gives the corresponding rates originating from the $h_{2} h_{125}$ state, assuming $\mathrm{BR}\left(h_{2} \rightarrow h_{125} h_{125}\right) \approx 68 \%$

\begin{tabular}{lllllll}
\hline $\mathrm{BR}\left(X \rightarrow F_{\mathrm{SM}}^{6}\right)$ & $6 b(\%)$ & $4 b 2 W(\%)$ & $2 b 4 W(\%)$ & $4 b 2 \tau(\%)$ & $4 b 2 Z(\%)$ & $4 b 2 \gamma(\%)$ \\
\hline$h_{125} h_{125} h_{125}$ & 20 & 22 & 7.8 & 6.6 & 2.8 & 0.24 \\
$h_{2} h_{125}$ & 14 & 15 & 5.3 & 4.5 & 1.9 & 0.16 \\
\hline
\end{tabular}




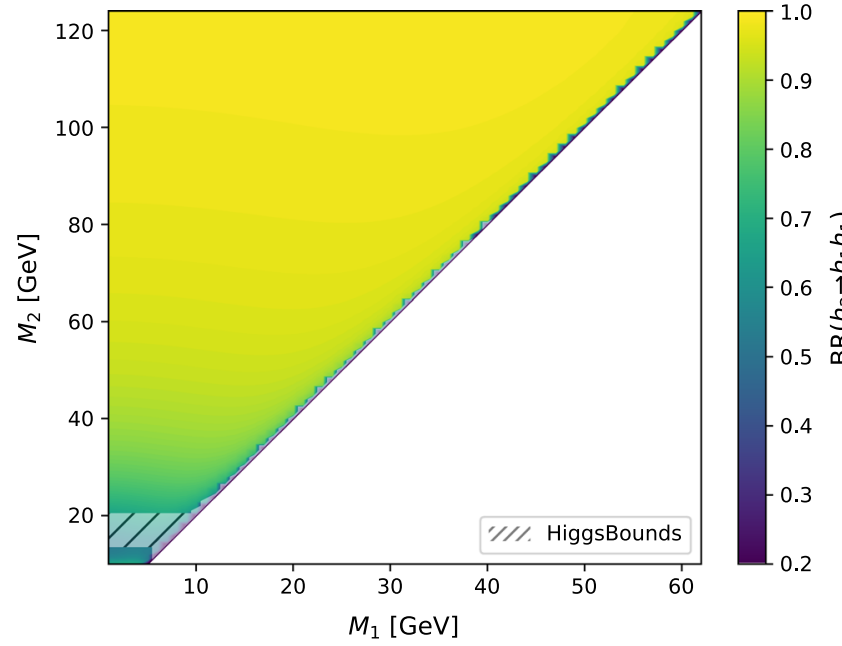

Fig. 11 Benchmark plane BP4 for the decay signature $h_{2} \rightarrow h_{1} h_{1}$ with $h_{125} \equiv h_{3}$, defined in the $\left(M_{1}, M_{2}\right)$ plane. The color code shows $\mathrm{BR}\left(h_{2} \rightarrow h_{1} h_{1}\right)$ (left panel) and the signal rate for $p p \rightarrow h_{2} \rightarrow h_{1} h_{1}$

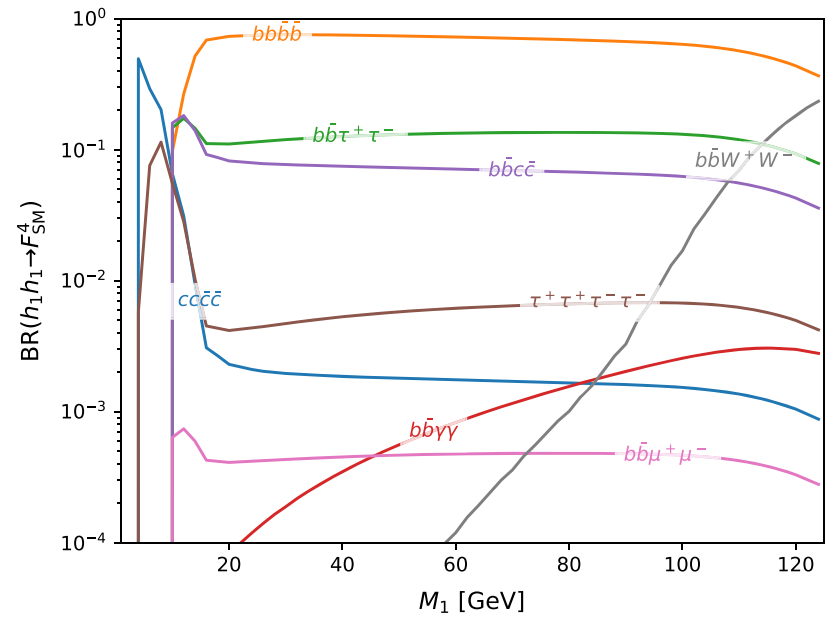

Fig. 12 Branching ratios of the $h_{1} h_{1}$ state of BP4 and BP5 into selected SM decay modes as a function of $M_{1}$

into $b \bar{b} b \bar{b}$ is dominant, followed by $b \bar{b} \tau^{+} \tau^{-}$. For even lighter $M_{1}$ the predominant decay is into charm quarks.

The $h_{125} h_{1} h_{1}$ coupling is very small in this scenario. Still - due to the large $\kappa_{125}$ - the process $p p \rightarrow h_{125} \rightarrow h_{1} h_{1}$ can enhance the total $h_{1} h_{1}$ production cross section by up to $\sim 15 \%$ for large $M_{2} \sim 125 \mathrm{GeV}$. On the other hand, interference effects between the resonant $h_{2}$ and $h_{125}$ contributions - similar to those discussed in Ref. [119] - remain negligible.

\subsection{BP5: $h_{3} \rightarrow h_{1} h_{1}$ with $h_{125} \equiv h_{2}$}

In the benchmark plane BP5 we identify $h_{125} \equiv h_{2}$ and consider the production of the heavier scalar $h_{3}$ followed by its symmetric decay to the lightest scalar, $h_{3} \rightarrow h_{1} h_{1}$. In our

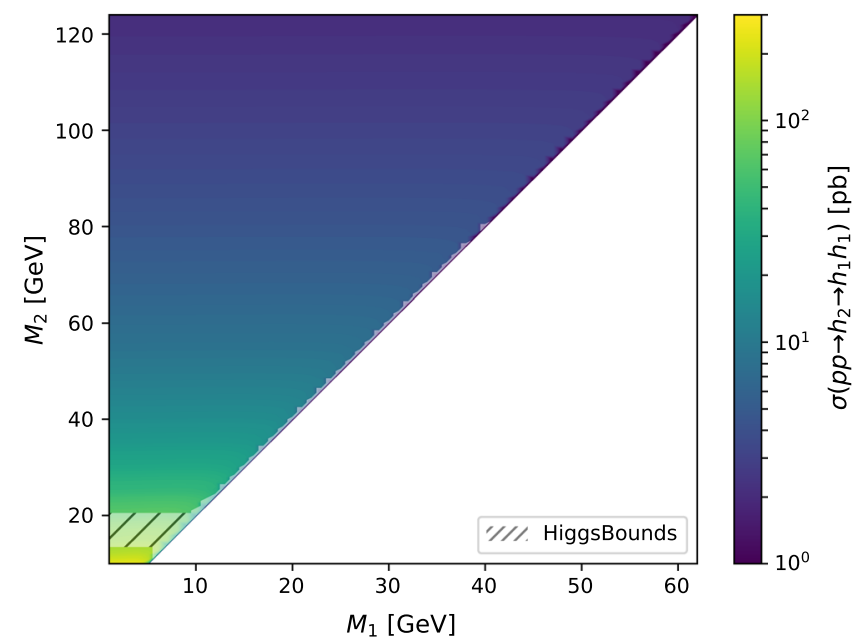

(right panel). The shaded region is excluded by LEP searches for $e^{+} e^{-} \rightarrow Z h_{2} \rightarrow Z(b \bar{b})[120]$

parameter scan of the TRSM (see Sect. 3.3) we found that parameter points exhibiting a sizeable $p p \rightarrow h_{3} \rightarrow h_{1} h_{1}$ rate also tend to be strongly constrained by the Higgs signal strength measurements if $2 M_{1}<125 \mathrm{GeV}$. In addition, if kinematically accessible, the decay modes $h_{3} \rightarrow h_{2} h_{2}$ and/or $h_{3} \rightarrow h_{1} h_{2}$ tend to dominate over the decay $h_{3} \rightarrow$ $h_{1} h_{1}$. In order to define a suitable benchmark scenario for the $p p \rightarrow h_{3} \rightarrow h_{1} h_{1}$ process it is therefore necessary that all triple Higgs couplings except for $\tilde{\lambda}_{113}$ are small while not overly suppressing $\kappa_{3}$. The chosen parameter values of BP5 are given in Table 2.

The phenomenology of BP5 is shown in Fig. 13. Throughout the parameter plane BR $\left(h_{3} \rightarrow h_{1} h_{1}\right)$ - shown in Fig. 13 (left) - exceeds $85 \%$ and approaches $100 \%$ for low values of $M_{3}$. The heavy scalar $h_{3}$ is produced at a rate of around $\kappa_{3}^{2} \simeq 6 \%$ of the corresponding prediction for a SM Higgs boson. Figure 13 (right) shows the resulting signal rates of $\mathcal{O}(0.1-1 \mathrm{pb})$ with maximal values of around $3 \mathrm{pb}$ for light $M_{3} \lesssim 150 \mathrm{GeV}$. The parameter region at $M_{1} \gtrsim 120 \mathrm{GeV}$ and $M_{3} \gtrsim 350 \mathrm{GeV}$ is constrained by LHC Higgs searches for resonant double Higgs production $[63,66]$. These are applied under the assumption that $h_{1}$ cannot be experimentally distinguished from $h_{125} \equiv h_{2}$ if they are close in mass and thus contributes to the predicted signal rate for this process.

The BRs of the $h_{1} h_{1}$ two-particle state can again be found in Fig. 12. They are identical to those discussed for BP4 since the BRs of $h_{1}$ are always identical to those of a SM Higgs boson of the same mass (see Sect. 2.2). However, now the scenario extends to $M_{1}$ values up to $125 \mathrm{GeV}$ and with increasing $M_{1}$ the final state $b \bar{b} W^{+} W^{-}$becomes sizable. In contrast to BP4, the two $h_{1}$ may be boosted if $M_{3} \gg 2 M_{1}$, 


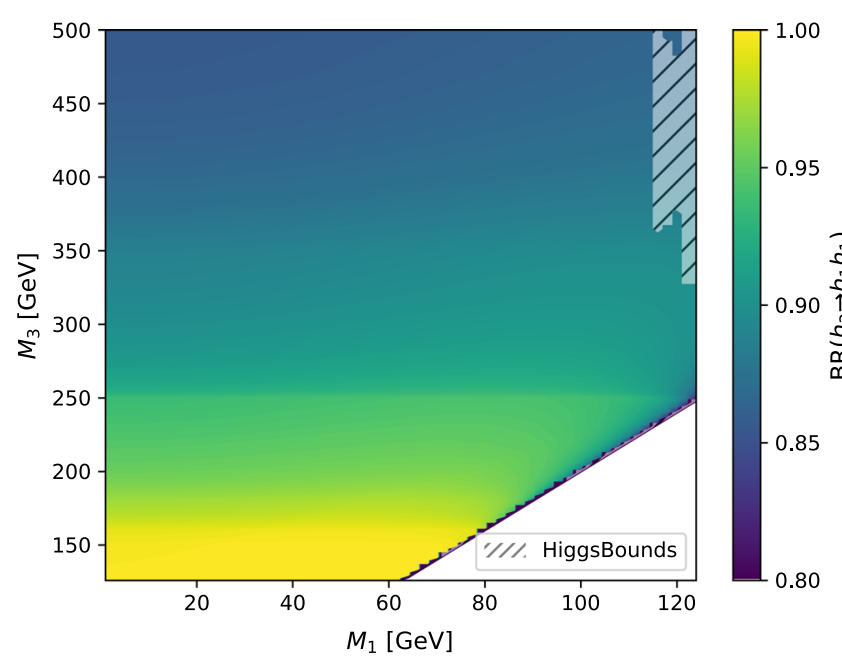

Fig. 13 Benchmark plane BP5 for the decay signature $h_{3} \rightarrow h_{1} h_{1}$ with $h_{125} \equiv h_{2}$, defined in the $\left(M_{1}, M_{3}\right)$ plane. The color code shows $\mathrm{BR}\left(h_{3} \rightarrow h_{1} h_{1}\right)$ (left panel) and the signal rate for $p p \rightarrow h_{3} \rightarrow h_{1} h_{1}$

leading to collimated $h_{1}$ decay products. This may provide an additional experimental handle, enabling the reduction of combinatoric background, and leading to a potential increase of the trigger sensitivity as well as the applicability of jet substructure techniques. Indeed, a recent ATLAS search for highly collimated photon-jets [123] probes the signature $p p \rightarrow h_{3} \rightarrow h_{1} h_{1} \rightarrow \gamma \gamma \gamma \gamma$ in the mass range $M_{3} \geq 200 \mathrm{GeV}, 0.1 \mathrm{GeV} \leq M_{1} \leq 10 \mathrm{GeV}$. However, the currently obtained limit is still several orders of magnitude larger than the predicted rate in BP5.

\subsection{BP6: $h_{3} \rightarrow h_{2} h_{2}$ with $h_{125} \equiv h_{1}$}

In benchmark plane BP6 we identify $h_{125} \equiv h_{1}$ and consider the production of the heaviest scalar $h_{3}$ followed by its symmetric decay $h_{3} \rightarrow h_{2} h_{2}$. This constrains the mass range for $h_{3}$ to values $M_{3}>250 \mathrm{GeV}$. This, in combination with the suppression of $\kappa_{3}$ due to the sum rule, Eq. (18), leads to relatively low production cross sections. The input parameters for BP6 are listed in Table 2.

Figure 14 shows the resulting $\left(M_{2}, M_{3}\right)$ parameter plane. The decay channel $h_{3} \rightarrow h_{2} h_{2}-$ shown in Fig. 14 (left) is the dominant decay mode of $h_{3}$ over the entire accessible parameter range with a $\mathrm{BR} \gtrsim 75 \%$ except close to the kinematic threshold. The heavy scalar $h_{3}$ is produced with about $\kappa_{3}^{2}=6 \%$ of the corresponding SM predicted rate. The resulting signal cross section in Fig. 14 (right) reaches $\sim 0.5 \mathrm{pb}$ in the low mass range, $M_{3} \lesssim 400 \mathrm{GeV}$, where $h_{2}$ decays directly to SM particles. The signal rates in the mass range $M_{3} \gtrsim 600 \mathrm{GeV}$, which is interesting for cascade decays, can reach up to $100 \mathrm{fb}$ for $p p \rightarrow h_{3} \rightarrow h_{2} h_{2}$ at the $13 \mathrm{TeV}$ LHC. In BP6 the total width of of $h_{3}$ can reach up to $\Gamma_{3} / M_{3} \sim 14 \%$ without violating the unitarity constraint. Therefore, it may

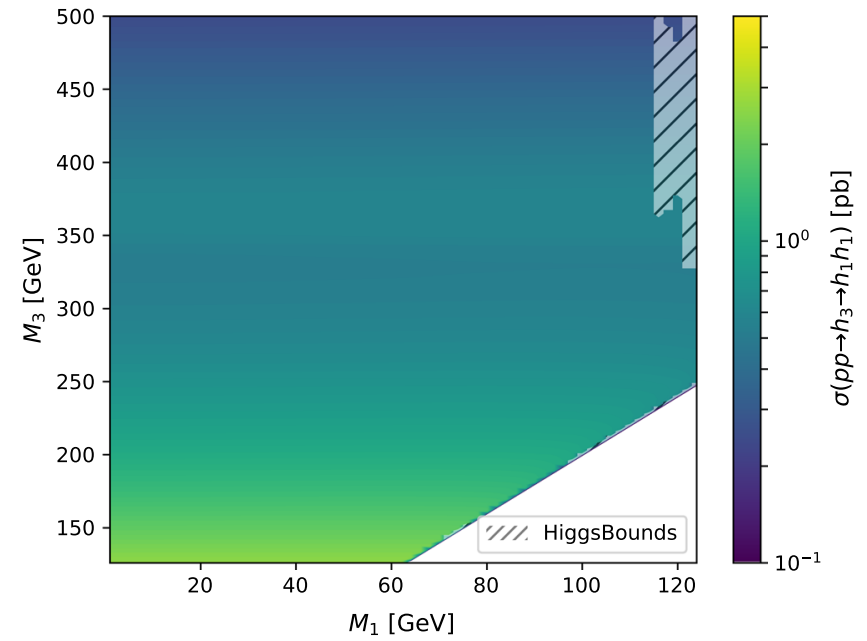

(right panel). The shaded region is excluded by searches for resonant double Higgs production $[63,66]$ via HiggsBounds

be important to include finite width effects in experimental analyses of this scenario.

The shaded region at large masses, $M_{3} \gtrsim 800 \mathrm{GeV}$, indicates that the parameter region is in conflict with perturbative unitarity. Additionally, experimental searches [66] are beginning to probe the region $M_{2} \sim 125 \mathrm{GeV}$. Similar to the discussion of BP5, this is a limit on $h_{3} \rightarrow h_{125} h_{125}$ which is sensitive under the assumption that $h_{2}$ and $h_{1} \equiv h_{125}$ cannot be experimentally distinguished if they are close in mass. Moreover, a first ATLAS search for the signature $p p \rightarrow h_{3} \rightarrow h_{2} h_{2} \rightarrow W^{+} W^{-} W^{+} W^{-}$[61] constrains a small region around $M_{2} \simeq 160 \mathrm{GeV}, M_{3} \simeq 330 \mathrm{GeV}$, as shown in Fig. 14. We expect this search analysis to sensitively probe this benchmark scenario in the future, in particular, if the currently considered mass range is extended. The ATLAS search only considers $h_{3}$ masses up to the $t \bar{t}$ threshold, $M_{3} \leq 340 \mathrm{GeV}$. However, as we discuss here, the $W^{+} W^{-} W^{+} W^{-}$final state remains the dominant fourparticle SM final state even beyond the $t \bar{t}$ threshold.

Figure 15 shows the BRs of the decays of the $h_{2} h_{2}$ state resulting from the $h_{3}$ decay in BP6. At low $M_{2}<250 \mathrm{GeV}$ only $h_{2} \rightarrow F_{\mathrm{SM}}$ decays are kinematically allowed. As shown in Fig. 15 (left), the dominant final state is $W^{+} W^{-} W^{+} W^{-}$, followed by $b \bar{b} W^{+} W^{-}$at low masses $M_{2} \lesssim 160 \mathrm{GeV}$ and $W^{+} W^{-} Z Z$ at larger mass values.

For $M_{2} \gtrsim 250 \mathrm{GeV}$ the branching ratio for $h_{2} \rightarrow$ $h_{125} h_{125}$ is about $40 \%$ and all three classes of decay chains from Fig. 5 can occur in BP6: direct decays of $h_{2} h_{2} \rightarrow F_{\mathrm{SM}}$; single cascade decays $h_{2} h_{2} \rightarrow h_{125} h_{125} h_{2} \rightarrow F_{\mathrm{SM}}$; and double cascade decays $h_{2} h_{2} \rightarrow h_{125} h_{125} h_{125} h_{125} \rightarrow F_{\mathrm{SM}}$, where the latter leads to a spectacular final state composed of four $h_{125}$. The BRs for direct decays of $h_{2} h_{2}$ to fourparticle $F_{\mathrm{SM}}$ are given in Fig. 15 (left). The dominant final 


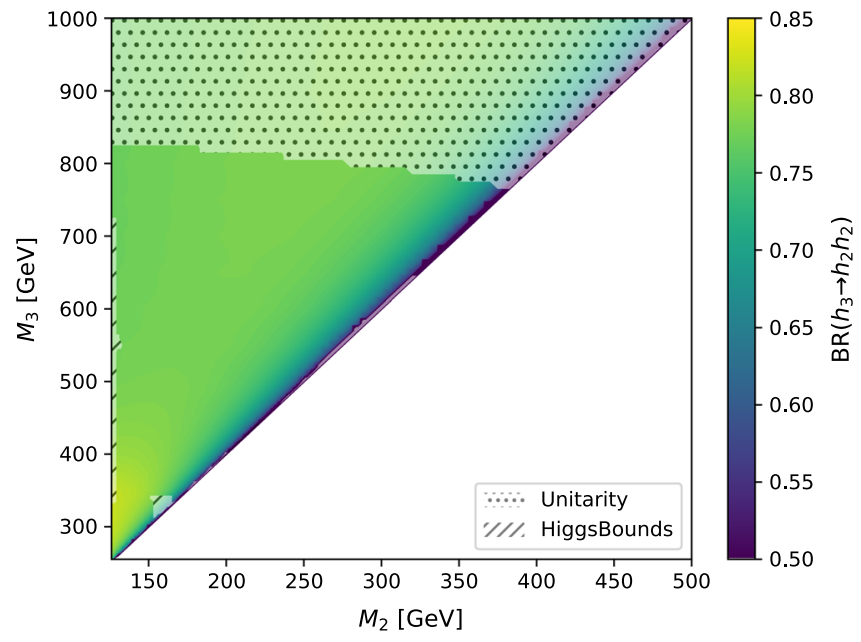

Fig. 14 Benchmark plane BP6 for the decay signature $h_{3} \rightarrow h_{2} h_{2}$ with $h_{125} \equiv h_{1}$, defined in the $\left(M_{2}, M_{3}\right)$ plane. The color code shows $\mathrm{BR}\left(h_{3} \rightarrow h_{2} h_{2}\right)$ (left panel) and the signal rate for $p p \rightarrow h_{3} \rightarrow h_{2} h_{2}$ (right panel). The shaded region at large $M_{3}$ is excluded by perturbative

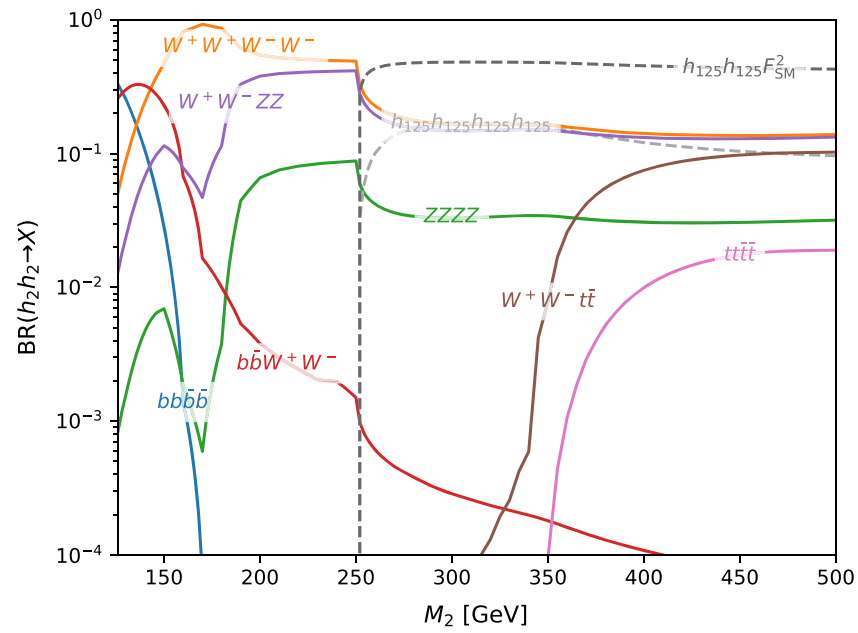

Fig. 15 Branching ratios of the $h_{2} h_{2}$ state of BP6. The left panel contains a selection of final states from direct decays of $h_{2} \rightarrow F_{\mathrm{SM}}$ and (inclusive) decays involving $h_{2} \rightarrow h_{125} h_{125}$ (both single and double

states of this class are $W^{+} W^{-} W^{+} W^{-}$and $W^{+} W^{-} Z Z$, with $W^{+} W^{-} t \bar{t}$ becoming comparable at high $M_{2}$. Figure 15 (left) also shows the "inclusive" branching ratio for the single cascade $h_{125} h_{125} F_{\mathrm{SM}}$ (summed over all possible $h_{2} \rightarrow F_{\mathrm{SM}}$ ) and the double cascade decay rate to the $h_{125} h_{125} h_{125} h_{125}$ final state.

The branching ratios of $h_{2} h_{2}$ into various six-particle SM final states via the single cascade decay are shown in Fig. 15 (right) as a function of $M_{2}$. The most important decay modes involve $b$ quarks and $W$ bosons and - due to combinatorial enhancement - have decay rates comparable to the four-particle final states. The decay $h_{2} h_{2} \rightarrow h_{125} h_{125} h_{2} \rightarrow$ $b \bar{b} b \bar{b} W^{+} W^{-}$is the third most likely decay mode of $h_{2} h_{2}$ for $250 \mathrm{GeV}<M_{2}<350 \mathrm{GeV}$.

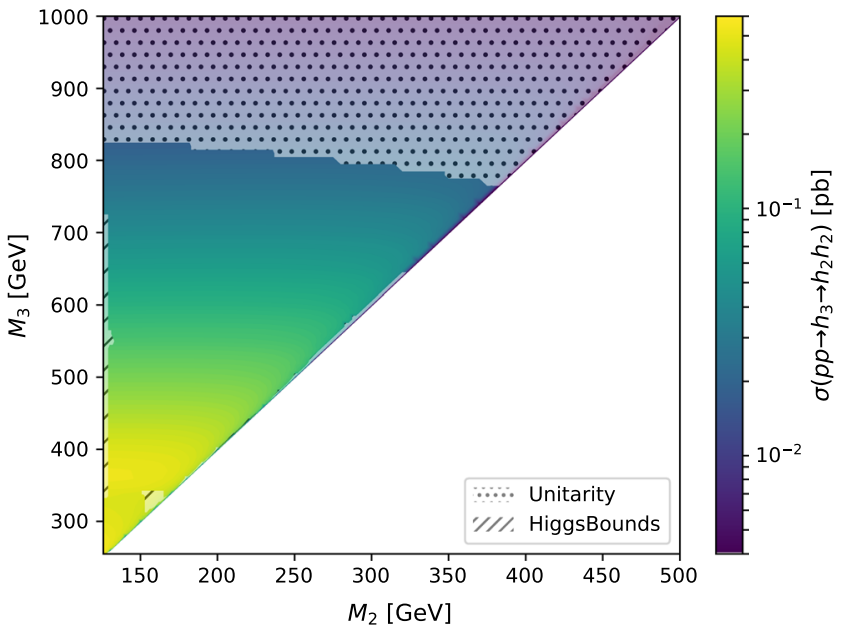

unitarity. The shaded region at $M_{2} \sim 125 \mathrm{GeV}$ is excluded by searches for resonant double Higgs production [66], and the shaded parameter region around $M_{2} \simeq 160 \mathrm{GeV}, M_{3} \simeq 330 \mathrm{GeV}$ by an ATLAS search for $h_{3} \rightarrow h_{2} h_{2} \rightarrow W^{+} W^{-} W^{+} W^{-}$[61] via HiggsBounds

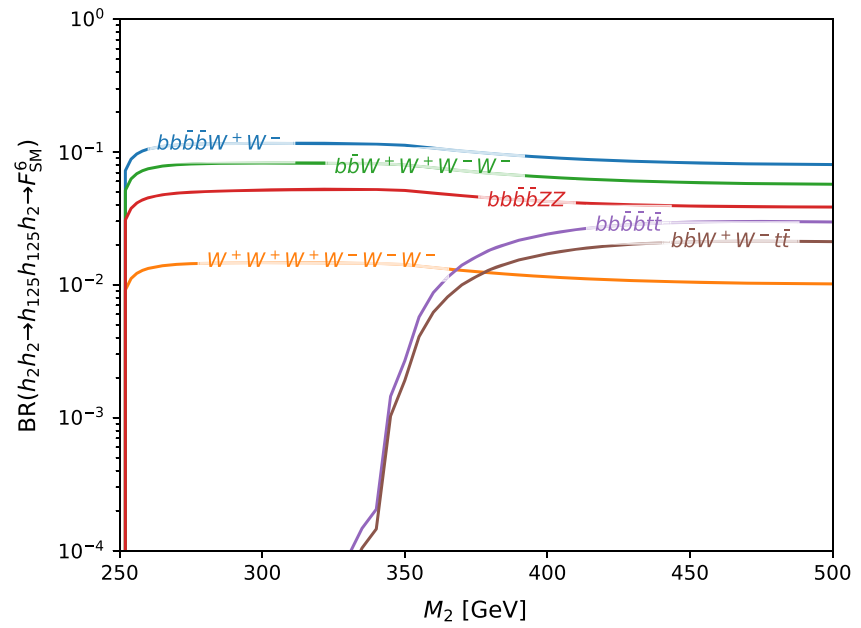

cascade). The right panel shows the most important six particle SM final states, $F_{\mathrm{SM}}^{6}$, that originate from a single cascade $h_{2} h_{2} \rightarrow h_{125} h_{125} F_{\mathrm{SM}}$

The branching ratios of the $h_{125} h_{125} h_{125} h_{125} \rightarrow F_{\mathrm{SM}}^{8}$ decays via a double cascade are independent of the model parameters. They are given in Table 4 . Since the BR for the double cascade shown in Fig. 15 (left) is almost independent of $M_{2}$ we include in the second row of Table 4 an approximate branching ratio for the decay of $h_{2} h_{2}$ into an eight-particle $\mathrm{SM}$ final state through the double cascade. For this we use the averaged $\operatorname{BR}\left(h_{2} h_{2} \rightarrow h_{125} h_{125} h_{125} h_{125}\right)=14.5 \%$, evaluated in the mass range $260 \mathrm{GeV}<M_{2}<500 \mathrm{GeV}$. The most important eight-particle final states are all combinations of decays into $b$ quarks and $W$ bosons - the most likely decay products of $h_{125}$. Due to combinatorial factors their overall branching fractions are, again, in some cases comparable to the four- and six-particle final states. For example, 
Table 4 Decay rates of the $h_{125} h_{125} h_{125} h_{125}$ state in BP6, leading to a eight-particle SM final state, $F_{\mathrm{SM}}^{8}$. The second row gives the corresponding rates originating from the $h_{2} h_{2}$ state, assuming $\operatorname{BR}\left(h_{2} h_{2} \rightarrow h_{125} h_{125} h_{125} h_{125}\right)=14.5 \%$

\begin{tabular}{lllllllll}
\hline $\mathrm{BR}\left(X \rightarrow F_{\mathrm{SM}}^{8}\right)$ & $6 b 2 W(\%)$ & $8 b(\%)$ & $4 b 4 W(\%)$ & $4 b 2 W 2 \tau(\%)$ & $6 b 2 \tau(\%)$ & $4 b 2 W 2 Z(\%)$ & $6 b 2 Z(\%)$ & $6 b 2 \gamma(\%)$ \\
\hline$h_{125} h_{125} h_{125} h_{125}$ & 17 & 12 & 9.2 & 5.5 & 5.2 & 2.3 & 2.2 & 0.19 \\
$h_{2} h_{2}$ & 2.5 & 1.8 & 1.3 & 0.8 & 0.75 & 0.34 & 0.31 & 0.027 \\
\hline
\end{tabular}

the $b \bar{b} b \bar{b} b \bar{b} W^{+} W^{-}$is similar to the $Z Z Z Z$ final state rate for masses $M_{3} \sim 300-350 \mathrm{GeV}$. Near the kinematic threshold, $M_{3} \gtrsim 500 \mathrm{GeV}$ and $M_{2} \gtrsim 250 \mathrm{GeV}$, the signal cross section for $p p \rightarrow h_{3} \rightarrow h_{2} h_{2} \rightarrow h_{125} h_{125} h_{125} h_{125}$ amounts to around $14 \mathrm{fb}$.

\section{Conclusion}

We presented the collider phenomenology of a simple extension of the SM Higgs sector, where two real scalar singlet fields are added to the particle content. In this two-realsinglet model we imposed a discrete $\mathbb{Z}_{2}$ symmetry for each scalar singlet field that is spontaneously broken by the singlet field's vacuum expectation value. Consequently, all scalar fields mix, leading to three neutral CP-even Higgs states $h_{a}$ $(a=1,2,3)$. Any of these states can be identified with the Higgs boson with mass $\simeq 125 \mathrm{GeV}$ observed at the LHC.

The model leads to an interesting collider phenomenology for searches for the additional Higgs states. Following the single production of one of the Higgs states, $h_{a}$, this state can either decay directly to SM particles, or it can decay into two lighter Higgs states, $h_{a} \rightarrow h_{b} h_{c}$, where the lighter states can either be identical ("symmetric" Higgs-to-Higgs decays with $b=c=1,2$ ), or different ("asymmetric" Higgs-toHiggs decays with $b=1, c=2$ ). In the latter case, successive decays of the second lightest Higgs state to the lightest Higgs state, $h_{2} \rightarrow h_{1} h_{1}$ may be possible if kinematically allowed. This leads to interesting Higgs-to-Higgs cascade decay signatures, in particular, $h_{3} \rightarrow h_{1,2} h_{2} \rightarrow F_{\mathrm{SM}} h_{1} h_{1}$ ("single cascade") and $h_{3} \rightarrow h_{2} h_{2} \rightarrow h_{1} h_{1} h_{1} h_{1}$ ("double cascade"), as shown in Fig. 5. We find that rates for all these possible Higgs-to-Higgs decays can in general be sizable, easily dominating the direct decay modes to SM particles.

Many of these Higgs-to-Higgs decay signatures have not been investigated experimentally to date. We therefore presented six two-dimensional benchmark scenarios to facilitate the design of dedicated experimental searches. Each scenario is defined such that one of the novel signatures has a nearly-maximal signal rate, while still obeying all theoretical and experimental constraints on the model. Moreover, as the model can be parametrized conveniently in terms of the relevant physical parameters, i.e. the three Higgs masses, three mixing angles (governing the Higgs coupling strengths to SM particles) and the three vevs, the benchmark scenarios can cover the entire kinematical phase space for the decay signatures, thus rendering them as ideal references for experimental searches.

For each benchmark scenario, we discussed in detail the rates of the relevant decays, as well as the expected signal rates in the TRSM at the $13 \mathrm{TeV}$ LHC. We furthermore provided an overview of the most relevant SM particle final states, as a function of the relevant mass parameters. This should provide a first step for experimental analyses to estimate the discovery potential of corresponding searches. We expect that some of the presented signatures can already be probed sensitively at the LHC with the data of $\sim 150 \mathrm{fb}^{-1}$ per experiment collected during Run-II.

It should be kept in mind that the Higgs-to-Higgs decay signatures (and potentially also the cascade decays) discussed here can generically appear also in other BSM models that feature three (or more) Higgs states. In that case, however, the Higgs coupling properties do not necessarily agree with those of the TRSM. This may result in different production rates of the resonantly-produced Higgs state, as well as different decay rates, in particular concerning the Higgs decays to SM particles. It is therefore important that future experimental searches present their results as limits - or ideally measurements - of the model-independent signal rate, as a function of the relevant kinematical quantities (Higgs masses and, possibly, total widths). Furthermore, Higgs-toHiggs decays to possible SM particle final states that are not dominant in the TRSM may still be worthwhile to probe experimentally, as the anticipated rates may be different in other models. In the case of a future discovery of an additional scalar state, signal rate measurements in various complementary production and decay modes will be crucial to probe its coupling structure and, in turn, to discriminate between the possible BSM interpretations.

The exploration of the scalar sector - leading to a better understanding of the mechanism of electroweak symmetry breaking - is one of the most important scientific goals of the LHC program. This endeavor requires an open-minded and unbiased view on the potential collider signatures of new scalars. Our discussion of the TRSM and the presented benchmark scenarios demonstrate that there is a plethora of currently unexplored collider signatures involving Higgs-toHiggs decays, and we hope that this work will initiate and facilitate the design of corresponding LHC searches in the near future. 
Acknowledgements We thank the LHC Higgs Cross Section Working Group WG3 conveners for encouraging this study, as well as Massimiliano Grazzini and Georg Weiglein for useful comments. We also thank Claudia Seitz for helpful discussions regarding experimental questions and Liang Li for helpful explanations regarding the experimental search presented in Ref. [61]. The work of TS and JW is funded by the Deutsche Forschungsgemeinschaft (DFG, German Research Foundation) under Germany's Excellence Strategy - EXC 2121 "Quantum Universe" 390833306. TR was supported by the European Union through the Programme Horizon 2020 via the COST action CA15108-Connecting insights in fundamental physics (FUNDAMENTALCONNECTIONS), and additionally wants to thank the DESY Theory group for repeated hospitality while this work was completed.

Data Availability Statement This manuscript has no associated data or the data will not be deposited. [Authors' comment: Data files for the benchmark scenarios can be provided by the authors upon request.]

Open Access This article is licensed under a Creative Commons Attribution 4.0 International License, which permits use, sharing, adaptation, distribution and reproduction in any medium or format, as long as you give appropriate credit to the original author(s) and the source, provide a link to the Creative Commons licence, and indicate if changes were made. The images or other third party material in this article are included in the article's Creative Commons licence, unless indicated otherwise in a credit line to the material. If material is not included in the article's Creative Commons licence and your intended use is not permitted by statutory regulation or exceeds the permitted use, you will need to obtain permission directly from the copyright holder. To view a copy of this licence, visit http://creativecomm ons.org/licenses/by/4.0/.

Funded by SCOAP ${ }^{3}$.

\section{Appendix A: Comparison of the TRSM with the complex scalar singlet extension}

The most general renormalizable and gauge invariant scalar potential of two real singlet fields $S$ and $X$ and the SM Higgs doublet $\Phi$ is

$$
\begin{aligned}
V= & \mu_{\Phi}^{2} \Phi^{\dagger} \Phi+\lambda_{\Phi}\left(\Phi^{\dagger} \Phi\right)^{2} \\
& +\mu_{S}^{2} S^{2}+\lambda_{S} S^{4}+\mu_{X}^{2} X^{2}+\lambda_{X} X^{4} \\
& +\lambda_{\Phi S} \Phi^{\dagger} \Phi S^{2}+\lambda_{\Phi X} \Phi^{\dagger} \Phi X^{2}+\lambda_{S X} S^{2} X^{2} \\
& +a_{S} S+a_{X} X+m_{S X} S X \\
& +T_{S S S} S^{3}+T_{X X X} X^{3}+T_{S S X} S^{2} X+T_{S X X} S X^{2} \\
& +T_{\Phi \Phi S} \Phi^{\dagger} \Phi S+T_{\Phi \Phi X} \Phi^{\dagger} \Phi X \\
& +\lambda_{S S S X} S^{3} X+\lambda_{S X X X} S X^{3}+\lambda_{\Phi \Phi S X} \Phi^{\dagger} \Phi S X
\end{aligned}
$$

The first three lines correspond to the TRSM scalar potential, Eq. (5), while the remaining lines break the $\mathbb{Z}_{2}$ symmetries of Eq. (4). These 21 real parameters relate to the 21 real parameters of the most general complex singlet extension, Eq. (1) of Ref. [33] (using the same notation), via

$$
\mu_{\Phi}^{2}=\frac{m^{2}}{2}, \quad \lambda_{\Phi}=\frac{\lambda}{4},
$$

$$
\begin{aligned}
\mu_{S}^{2} & =\frac{1}{2}\left(b_{2}+b_{1} \cos \phi_{b_{1}}\right), \\
\lambda_{S} & =\frac{1}{4}\left(d_{1} \cos \phi_{d_{1}}+d_{3} \cos \phi_{d_{3}}+d_{2}\right), \\
\mu_{X}^{2} & =\frac{1}{2}\left(b_{2}-b_{1} \cos \phi_{b_{1}}\right), \\
\lambda_{X} & =\frac{1}{4}\left(d_{1} \cos \phi_{d_{1}}-d_{3} \cos \phi_{d_{3}}+d_{2}\right), \\
\lambda_{\Phi S} & =\frac{1}{2}\left(\delta_{2}+\delta_{3} \cos \phi_{\delta_{3}}\right), \\
\lambda_{\Phi X} & =\frac{1}{2}\left(\delta_{2}-\delta_{3} \cos \phi_{\delta_{3}}\right), \\
\lambda_{S X} & =-\frac{3}{2} d_{1} \cos \phi_{d_{1}}+\frac{d_{2}}{2}, \\
a_{S} & =2 a_{1} \cos \phi_{a_{1}}, \quad a_{X}=-2 a_{1} \sin \phi_{a_{1}} \\
m_{S X} & =-b_{1} \sin \phi_{b_{1}}, \\
T_{S S S} & =\frac{1}{3}\left(c_{1} \cos \phi_{c_{1}}+c_{2} \cos \phi_{c_{2}}\right) \\
T_{X X X} & =\frac{1}{3}\left(c_{1} \sin \phi_{c_{1}}-c_{2} \sin \phi_{c_{2}}\right), \\
T_{S S X} & =-c_{1} \sin \phi_{c_{1}}-\frac{c_{2}}{3} \sin \phi_{c_{2}}, \\
T_{S X X} & =-c_{1} \cos \phi_{c_{1}}+\frac{c_{2}}{3} \cos \phi_{c_{2}}, \\
T_{\Phi \Phi S} & =\frac{\delta_{1}}{2} \cos \phi_{\delta_{1}}, \quad T_{\Phi \Phi X}=-\frac{\delta_{1}}{2} \sin \phi_{\delta_{1}}, \\
\lambda_{S S S X} & =-d_{1} \sin \phi_{d_{1}}-\frac{d_{3}}{2} \sin \phi_{d_{3}}, \\
\lambda_{S X X X} & =d_{1} \sin \phi_{d_{1}}-\frac{d_{3}}{2} \sin \phi_{d_{3}}, \\
\lambda_{\Phi \Phi X} & =-\delta_{3} \sin \phi_{\delta_{3}} . \\
&
\end{aligned}
$$

Using these relations we could alternatively parametrize the TRSM as a complex singlet extension where

$$
\begin{aligned}
& a_{1}=c_{1}=c_{2}=\delta_{1}=0, \\
& \sin \phi_{b_{1}}=\sin \phi_{d_{1}}=\sin \phi_{d_{3}}=\sin \phi_{\delta_{3}}=0 .
\end{aligned}
$$

The $U(1)$ symmetry imposed on the complex scalar in Ref. [35] requires $\delta_{1}=\delta_{3}=c_{1}=c_{2}=d_{1}=b_{3}=a_{1}=b_{1}=0$. This forms a special case of the TRSM where

$\mu_{X}^{2}=\mu_{S}^{2}, \quad \lambda_{\Phi S}=\lambda_{\Phi X}, \quad \lambda_{S}=\lambda_{X}=\frac{1}{2} \lambda_{S X}$.

In this model soft $U(1)$ breaking terms - such as $a_{1}, b_{1} \neq 0$ are required to avoid a massless Goldstone boson. With these terms included, the resulting model is no longer a special case of the TRSM.

\section{References}

1. F. Englert, R. Brout, Phys. Rev. Lett. 13, 321 (1964)

2. P.W. Higgs, Phys. Lett. 12, 132 (1964) 
3. P.W. Higgs, Phys. Rev. Lett. 13, 508 (1964)

4. G.S. Guralnik, C.R. Hagen, T.W.B. Kibble, Phys. Rev. Lett. 13, 585 (1964)

5. P.W. Higgs, Phys. Rev. 145, 1156 (1966)

6. T.W.B. Kibble, Phys. Rev. 155, 1554 (1967)

7. G. Aad et al. (ATLAS), Phys. Lett. B 716, 1 (2012). arXiv: 1207.7214 [hep-ex]

8. S. Chatrchyan et al. (CMS), Phys. Lett. B 716, 30 (2012). arXiv:1207.7235 [hep-ex]

9. G. Aad et al. (ATLAS, CMS), JHEP 08, 045 (2016). arXiv:1606.02266 [hep-ex]

10. A.M. Sirunyan et al. (CMS), Phys. Lett. B 780, 501 (2018). arXiv: 1709.07497 [hep-ex]

11. A.M. Sirunyan et al. (CMS), Phys. Rev. Lett. 120, 071802 (2018). arXiv: 1709.05543 [hep-ex]

12. CMS collaboration, CMS-PAS-HIG-18-029 (2019)

13. CMS collaboration, CMS-PAS-HIG-18-030 (2019)

14. A.M. Sirunyan et al. (CMS), Phys. Rev. Lett. 122, 021801 (2019). arXiv:1807.06325 [hep-ex]

15. A.M. Sirunyan et al. (CMS), JHEP 08, 066 (2018). arXiv:1803.05485 [hep-ex]

16. A.M. Sirunyan et al. (CMS), Phys. Lett. B 791, 96 (2019). arXiv: 1806.05246 [hep-ex]

17. CMS Collaboration, CMS-PAS-HIG-18-019 (2018)

18. G. Aad et al. (ATLAS), Phys. Rev. D 101, 012002 (2020). arXiv:1909.02845 [hep-ex]

19. CMS collaboration, CMS-PAS-HIG-19-001 (2019)

20. CMS collaboration, CMS-PAS-HIG-18-032 (2019)

21. G.C. Branco, L. Lavoura, J.P. Silva, Int. Ser. Monogr. Phys. 103, 1 (1999)

22. I.P. Ivanov, Prog. Part. Nucl. Phys. 95, 160 (2017). arXiv:1702.03776 [hep-ph]

23. A. Datta, A. Raychaudhuri, Phys. Rev. D 57, 2940 (1998). arXiv:hep-ph/9708444 [hep-ph]

24. R.M. Schabinger, J.D. Wells, Phys. Rev. D 72, 093007 (2005). arXiv:hep-ph/0509209 [hep-ph]

25. B. Patt, F. Wilczek, (2006). arXiv:hep-ph/0605188 [hep-ph]

26. D. O'Connell, M.J. Ramsey-Musolf, M.B. Wise, Phys. Rev. D 75, 037701 (2007). arXiv:hep-ph/0611014 [hep-ph]

27. V. Barger, P. Langacker, M. McCaskey, M.J. Ramsey-Musolf, G. Shaughnessy, Phys. Rev. D 77, 035005 (2008). arXiv:0706.4311 [hep-ph]

28. C.-Y. Chen, S. Dawson, I.M. Lewis, Phys. Rev. D 91, 035015 (2015). arXiv:1410.5488 [hep-ph]

29. T. Robens, T. Stefaniak, Eur. Phys. J. C 75, 104 (2015). arXiv:1501.02234 [hep-ph]

30. T. Robens, T. Stefaniak, Eur. Phys. J. C 76, 268 (2016). arXiv:1601.07880 [hep-ph]

31. I.M. Lewis, M. Sullivan, Phys. Rev. D 96, 035037 (2017). arXiv:1701.08774 [hep-ph]

32. A. Ilnicka, T. Robens, T. Stefaniak, Mod. Phys. Lett. A 33, 1830007 (2018). arXiv:1803.03594 [hep-ph]

33. V. Barger, P. Langacker, M. McCaskey, M. Ramsey-Musolf, G. Shaughnessy, Phys. Rev. D 79, 015018 (2009). arXiv:0811.0393 [hep-ph]

34. L. Alexander-Nunneley, A. Pilaftsis, JHEP 09, 021 (2010). arXiv: 1006.5916 [hep-ph]

35. R. Coimbra, M.O.P. Sampaio, R. Santos, Eur. Phys. J. C 73, 2428 (2013). arXiv:1301.2599 [hep-ph]

36. A. Ahriche, A. Arhrib, S. Nasri, JHEP 02, 042 (2014). arXiv: 1309.5615 [hep-ph]

37. R. Costa, A.P. Morais, M.O.P. Sampaio, R. Santos, Phys. Rev. D 92, 025024 (2015). arXiv:1411.4048 [hep-ph]

38. R. Costa, M. Mühlleitner, M.O.P. Sampaio, R. Santos, JHEP 06, 034 (2016). arXiv:1512.05355 [hep-ph]
39. P.M. Ferreira, Phys. Rev. D 94, 096011 (2016). arXiv:1607.06101 [hep-ph]

40. J. Chang, K. Cheung, S.-C. Hsu, C.-T. Lu, Phys. Rev. D 95, 035012 (2017). arXiv:1607.07550 [hep-ph]

41. M. Mühlleitner, M.O.P. Sampaio, R. Santos, J. Wittbrodt, JHEP 08, 132 (2017). arXiv:1703.07750 [hep-ph]

42. S. Dawson, M. Sullivan, Phys. Rev. D 97, 015022 (2018). arXiv:1711.06683 [hep-ph]

43. R.N. Lerner, J. McDonald, Phys. Rev. D 80, 123507 (2009). arXiv:0909.0520 [hep-ph]

44. V. Barger, M. McCaskey, G. Shaughnessy, Phys. Rev. D 82, 035019 (2010). arXiv:1005.3328 [hep-ph]

45. M. Gonderinger, H. Lim, M.J. Ramsey-Musolf, Phys. Rev. D 86 , 043511 (2012). arXiv:1202.1316 [hep-ph]

46. G. Belanger, K. Kannike, A. Pukhov, M. Raidal, JCAP 1301, 022 (2013). arXiv:1211.1014 [hep-ph]

47. K. Ghorbani, H. Ghorbani, Phys. Rev. D 93, 055012 (2016). arXiv:1501.00206 [hep-ph]

48. M. Jiang, L. Bian, W. Huang, J. Shu, Phys. Rev. D 93, 065032 (2016). arXiv:1502.07574 [hep-ph]

49. P. Athron et al. (GAMBIT), Eur. Phys. J. C77, 568 (2017). arXiv:1705.07931 [hep-ph]

50. C.-W. Chiang, M.J. Ramsey-Musolf, E. Senaha, Phys. Rev. D 97, 015005 (2018). arXiv:1707.09960 [hep-ph]

51. P. Athron, J.M. Cornell, F. Kahlhoefer, J. Mckay, P. Scott, S. Wild, Eur. Phys. J. C 78, 830 (2018). arXiv:1806.11281 [hep-ph]

52. B. Grzadkowski, D. Huang, JHEP 08, 135 (2018). arXiv:1807.06987 [hep-ph]

53. D. Curtin, P. Meade, C.-T. Yu, JHEP 11, 127 (2014). arXiv:1409.0005 [hep-ph]

54. A.V. Kotwal, M.J. Ramsey-Musolf, J.M. No, P. Winslow, Phys. Rev. D 94, 035022 (2016). arXiv:1605.06123 [hep-ph]

55. A. Beniwal, M. Lewicki, J.D. Wells, M. White, A.G. Williams, JHEP 08, 108 (2017). arXiv:1702.06124 [hep-ph]

56. W. Cheng, L. Bian, Phys. Rev. D 98, 023524 (2018). arXiv:1801.00662 [hep-ph]

57. K. Ghorbani, P.H. Ghorbani, JHEP 12, 077 (2019). arXiv:1906.01823 [hep-ph]

58. M. Aaboud et al. (ATLAS), Phys. Rev. D 94, 052002 (2016). arXiv:1606.04782 [hep-ex]

59. A.M. Sirunyan et al. (CMS), JHEP 01, 054 (2018). arXiv: 1708.04188 [hep-ex]

60. A.M. Sirunyan et al. (CMS), Phys. Lett. B 778, 101 (2018). arXiv: 1707.02909 [hep-ex]

61. M. Aaboud et al. (ATLAS), JHEP 05, 124 (2019). arXiv:1811.11028 [hep-ex]

62. M. Aaboud et al. (ATLAS), JHEP 04, 092 (2019). arXiv:1811.04671 [hep-ex]

63. M. Aaboud et al. (ATLAS), Phys. Rev. Lett. 121, 191801 (2018), [Erratum: Phys. Rev. Lett.122,no.8,089901(2019)]. arXiv:1808.00336 [hep-ex]

64. M. Aaboud et al. (ATLAS), Eur. Phys. J. C 78, 1007 (2018). arXiv: 1807.08567 [hep-ex]

65. M. Aaboud et al. (ATLAS), JHEP 11, 040 (2018). arXiv: 1807.04873 [hep-ex]

66. A.M. Sirunyan et al. (CMS), Phys. Rev. Lett. 122, 121803 (2019). arXiv:1811.09689 [hep-ex]

67. A.M. Sirunyan et al. (CMS), JHEP 08, 152 (2018). arXiv: 1806.03548 [hep-ex]

68. A.M. Sirunyan et al. (CMS), Phys. Lett. B 788, 7 (2019). arXiv:1806.00408 [hep-ex]

69. G. Aad et al. (ATLAS), Phys. Lett. B800, 135103 (2020). arXiv:1906.02025 [hep-ex]

70. A.M. Sirunyan et al. (CMS), JHEP 11, 018 (2018). arXiv: 1805.04865 [hep-ex] 
71. A.M. Sirunyan et al. (CMS), Phys. Lett. B 785, 462 (2018). arXiv:1805.10191 [hep-ex]

72. M. Aaboud et al. (ATLAS), JHEP 10, 031 (2018). arXiv:1806.07355 [hep-ex]

73. M. Aaboud et al. (ATLAS), Phys. Lett. B 782, 750 (2018). arXiv: 1803.11145 [hep-ex]

74. A.M. Sirunyan et al. (CMS), Phys. Lett. B 795, 398 (2019). arXiv:1812.06359 [hep-ex]

75. M. Aaboud et al. (ATLAS), Phys. Lett. B 790, 1 (2019). arXiv: 1807.00539 [hep-ex]

76. A.M. Sirunyan et al. (CMS), Phys. Lett. B 800, 135087 (2020). arXiv:1907.07235 [hep-ex]

77. D. de Florian et al. (LHC Higgs Cross Section Working Group), (2016). https://doi.org/10.23731/CYRM-2017-002. arXiv:1610.07922 [hep-ph]

78. A. Djouadi, J. Kalinowski, M. Spira, Comput. Phys. Commun. 108, 56 (1998). arXiv:hep-ph/9704448 [hep-ph]

79. J.M. Butterworth et al., in Physics at TeV colliders. Proceedings, 6th Workshop, dedicated to Thomas Binoth, Les Houches, France, June 8-26, 2009 (2010). arXiv:1003.1643 [hep-ph]

80. A. Djouadi, J. Kalinowski, M. Muehlleitner, M. Spira, Comput. Phys. Commun. 238, 214 (2019). arXiv:1801.09506 [hep-ph]

81. A. Bredenstein, A. Denner, S. Dittmaier, M.M. Weber, Phys. Rev. D 74, 013004 (2006). arXiv:hep-ph/0604011 [hep-ph]

82. A. Bredenstein, A. Denner, S. Dittmaier, M.M. Weber, Proceedings, 8th DESY Workshop on Elementary Particle Theory: Loops and Legs in Quantum Field Theory: Eisenach, Germany, 23-28 April 2006, Nucl. Phys. Proc. Suppl. 160, 131 (2006), [131(2006)]. arXiv:hep-ph/0607060 [hep-ph]

83. A. Bredenstein, A. Denner, S. Dittmaier, M.M. Weber, JHEP 02, 080 (2007). arXiv:hep-ph/0611234 [hep-ph]

84. K. Kannike, Eur. Phys. J. C 72, 2093 (2012). arXiv:1205.3781 [hep-ph]

85. K. Kannike, Eur. Phys. J. C 76, 324 (2016), [Erratum: Eur. Phys. J.C78,no.5,355(2018)]. arXiv:1603.02680 [hep-ph]

86. G. Altarelli, R. Barbieri, Phys. Lett. B 253, 161 (1991)

87. M.E. Peskin, T. Takeuchi, Phys. Rev. Lett. 65, 964 (1990)

88. M.E. Peskin, T. Takeuchi, Phys. Rev. D 46, 381 (1992)

89. I. Maksymyk, C.P. Burgess, D. London, Phys. Rev. D 50, 529 (1994). arXiv:hep-ph/9306267 [hep-ph]

90. J. Haller, A. Hoecker, R. Kogler, K. Mönig, T. Peiffer, J. Stelzer, Eur. Phys. J. C 78, 675 (2018). arXiv:1803.01853 [hep-ph]

91. W. Grimus, L. Lavoura, O.M. Ogreid, P. Osland, J. Phys. G 35, 075001 (2008). arXiv:0711.4022 [hep-ph]

92. W. Grimus, L. Lavoura, O.M. Ogreid, P. Osland, Nucl. Phys. B 801, 81 (2008). arXiv:0802.4353 [hep-ph]

93. D. Lopez-Val, T. Robens, Phys. Rev. D 90, 114018 (2014). arXiv:1406.1043 [hep-ph]

94. S. Schael et al. (ALEPH, DELPHI, L3, OPAL, LEP Electroweak), Phys. Rept. 532, 119 (2013). arXiv:1302.3415 [hep-ex]

95. T.A. Aaltonen et al. (CDF), Phys. Rev. D 89, 072003 (2014). arXiv:1311.0894 [hep-ex]

96. M. Aaboud et al. (ATLAS), Eur. Phys. J. C 78, 110 (2018), [Erratum: Eur. Phys. J.C78,no.11,898(2018)]. arXiv:1701.07240 [hepex]

97. M. Tanabashi et al. (Particle Data Group), Phys. Rev. D 98, 030001 (2018)

98. P. Bechtle, O. Brein, S. Heinemeyer, G. Weiglein, K.E. Williams, Comput. Phys. Commun. 181, 138 (2010). arXiv:0811.4169 [hep$\mathrm{ph}]$

99. P. Bechtle, O. Brein, S. Heinemeyer, G. Weiglein, K.E. Williams, Comput. Phys. Commun. 182, 2605 (2011). arXiv:1102.1898 [hep-ph]
100. P. Bechtle, O. Brein, S. Heinemeyer, O. Stål, T. Stefaniak, G. Weiglein, K. Williams, Proceedings, 4th International Workshop on Prospects for Charged Higgs Discovery at Colliders (CHARGED 2012): Uppsala, Sweden, October 8-11, 2012, PoS, vol. CHARGED2012, p. 024 (2012). arXiv:1301.2345 [hep-ph]

101. P. Bechtle, O. Brein, S. Heinemeyer, O. Stål, T. Stefaniak, G. Weiglein, K.E. Williams, Eur. Phys. J. C 74, 2693 (2014). arXiv:1311.0055 [hep-ph]

102. P. Bechtle, S. Heinemeyer, O. Stål, T. Stefaniak, G. Weiglein, Eur. Phys. J. C 75, 421 (2015). arXiv:1507.06706 [hep-ph]

103. P. Bechtle, D. Dercks, S. Heinemeyer, T. Klingl, T. Stefaniak, G. Weiglein, J. Wittbrodt, "HiggsBounds," https://gitlab.com/ higgsbounds/higgsbounds (2019)

104. O. Stål, T. Stefaniak, Proceedings, 2013 European Physical Society Conference on High Energy Physics (EPS-HEP 2013): Stockholm, Sweden, July 18-24, 2013, PoS, vol. EPS-HEP2013. p. 314 (2013). arXiv:1310.4039 [hep-ph]

105. P. Bechtle, S. Heinemeyer, O. Stål, T. Stefaniak, G. Weiglein, Eur. Phys. J. C 74, 2711 (2014). arXiv:1305.1933 [hep-ph]

106. P. Bechtle, S. Heinemeyer, O. Stål, T. Stefaniak, G. Weiglein, JHEP 11, 039 (2014). arXiv:1403.1582 [hep-ph]

107. P. Bechtle, D. Dercks, S. Heinemeyer, T. Klingl, T. Stefaniak, G. Weiglein, J. Wittbrodt, "HiggsSignals". https://gitlab.com/ higgsbounds/higgssignals (2019)

108. ATLAS collaboration, ATLAS-CONF-2019-005 (2019)

109. M. Cepeda et al. (Physics of the HL-LHC Working Group) (2019). arXiv:1902.00134 [hep-ph]

110. A. M. Sirunyan et al. (CMS), Phys. Lett. B 793, 520 (2019). arXiv:1809.05937 [hep-ex]

111. M. Aaboud et al. (ATLAS), Phys. Rev. Lett. 122, 231801 (2019). arXiv:1904.05105 [hep-ex]

112. P.M. Ferreira, R. Guedes, M.O.P. Sampaio, R. Santos, JHEP 12, 067 (2014). arXiv:1409.6723 [hep-ph]

113. M. Mühlleitner, M.O.P. Sampaio, R. Santos, J. Wittbrodt, JHEP 03, 094 (2017). arXiv:1612.01309 [hep-ph]

114. J.R. Andersen et al. (LHC Higgs Cross Section Working Group), 10.5170/CERN-2013-004 (2013). arXiv:1307.1347 [hep-ph]

115. C. Anastasiou, C. Duhr, F. Dulat, E. Furlan, T. Gehrmann, F. Herzog, A. Lazopoulos, B. Mistlberger, JHEP 05, 058 (2016). arXiv:1602.00695 [hep-ph]

116. S. Dawson, I.M. Lewis, Phys. Rev. D 92, 094023 (2015). arXiv:1508.05397 [hep-ph]

117. M. Carena, Z. Liu, M. Riembau, Phys. Rev. D 97, 095032 (2018). arXiv:1801.00794 [hep-ph]

118. J. Alison et al., in Double Higgs Production at Colliders Batavia, IL, USA, September 4, 2018-9, 2019, ed. by B. Di Micco, M. Gouzevitch, J. Mazzitelli, C. Vernieri (2019). arXiv:1910.00012 [hep-ph]

119. P. Basler, S. Dawson, C. Englert, M.Mühlleitner, Phys. Rev. D 101, 015019 (2020). arXiv:1909.09987 [hep-ph]

120. S. Schael et al. (ALEPH, DELPHI, L3, OPAL, LEP Working Group for Higgs Boson Searches), Eur. Phys. J. C 47, 547 (2006). arXiv:hep-ex/0602042 [hep-ex]

121. M. Aaboud et al. (ATLAS), Phys. Rev. D 98, 052008 (2018). arXiv:1808.02380 [hep-ex]

122. A.M. Sirunyan et al. (CMS), JHEP 06, 127 (2018). [Erratum: JHEP03,128(2019)]. arXiv:1804.01939 [hep-ex]

123. M. Aaboud et al. (ATLAS), Phys. Rev. D 99, 012008 (2019). arXiv:1808.10515 [hep-ex] 